\title{
Itô calculus without probability in idealized financial markets
}

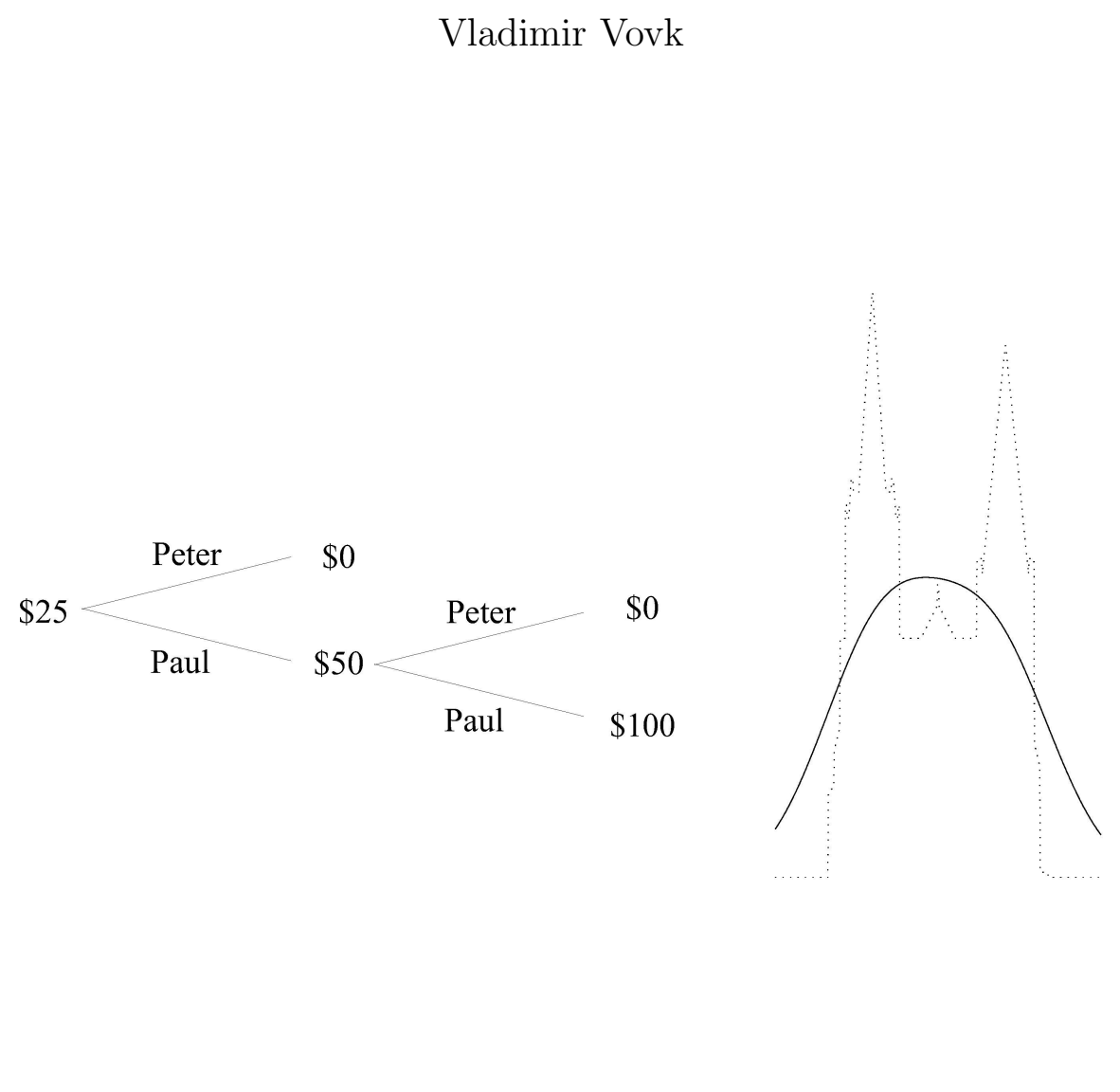

The Game-Theoretic Probability and Finance Project

Working Paper \#36

First posted August 3, 2011. Last revised October 25, 2018.

Project web site:

http://www.probabilityandfinance.com 


\begin{abstract}
We consider idealized financial markets in which price paths of the traded securities are càdlàg functions, imposing mild restrictions on the allowed size of jumps. We prove the existence of quadratic variation for typical price paths, where the qualification "typical" means that there is a trading strategy that risks only one monetary unit and brings infinite capital if quadratic variation does not exist. This result allows one to apply numerous known results in pathwise Itô calculus to typical price paths; we give a brief overview of such results.
\end{abstract}

\title{
Contents
}

1 Introduction 1

2 Pathwise quadratic variation 2

3 Typical price paths 4

4 Existence of quadratic variation 8

5 Multidimensional case 16

6 Föllmer's and Norvaiša's quadratic variation 19

7 Implications of the existence of quadratic variation 23

8 Conclusion 25

References $\quad[26$ 


\section{Introduction}

Itô calculus, based on the notion of the stochastic integral, plays an important role in mathematical finance. However, the usual construction of the stochastic integral relies on statistical assumptions about security prices, and it is not easy to come up with their realistic statistical models, as witnessed by the proliferation of various competing models in recent years. The pathwise stochastic integral and Itô calculus proposed in 1981 by Föllmer [6] and developed by numerous authors (see, e.g., [14, 3]) do not depend on any statistical assumptions. They, however, depend on the existence of quadratic variation for the allowed paths, which is postulated. The goal of this paper is to establish the existence of quadratic variation of security prices under weak conditions that can be justified from the economic point of view.

The existence of quadratic variation for typical continuous price paths was established in [21], where it served as a tool for studying properties of typical price paths, such as their volatility. This paper proves the existence of quadratic variation under a weaker assumption than in [21]: the price paths are assumed to be càdlàg without huge jumps. A related result was obtained in 22]: that paper shows that the $p$-variation of typical prices paths is finite for $p>2$. The assumptions of 22 are not comparable to the assumptions of this paper: on one hand, there are no restrictions on the size of jumps in 22, but on the other hand, the price paths are assumed nonnegative (albeit strong, this assumption is natural in the context of financial markets). In this paper, we will often be using results and methods of 22 .

This paper, like [21] and [22], is written in the tradition of game-theoretic probability (see, e.g., [17, 19, 11, 12, 13]). In game-theoretic probability, probability-like notions (such as the upper probabilities of events and upper prices of functions) are defined in terms of idealized financial markets, and mathematical finance is a natural field of application. The key technical tool used in this paper will be "high-frequency limit order strategies", introduced in game-theoretic probability by 19 .

We start the main part of the paper by defining in Section 2 the notion of quadratic variation, a version of Föllmer's pathwise definition adapted to our goals. The next section, Section 3, states and discusses our assumption about the jumps of price paths and defines the notion of a typical price path. Our main result, the existence of quadratic variation for typical price paths, is established in Section 4. Section 5 extends this result to typical vector-valued price paths; in particular, it proves the existence of quadratic covariation between two price paths. Section 6 compares the notion of quadratic variation used in this paper with Norvaiša's [14] and Föllmer's [6]; we show that under natural conditions (satisfied in the main example in this paper) the first two notions of quadratic variation are equivalent, whereas Föllmer's notion becomes equivalent to them when another natural condition is added. Section 7 summarizes some of the known results for price paths possessing quadratic variation. 


\section{Notation}

The set of all real (resp. rational, resp. integer) numbers is denoted $\mathbb{R}$ (resp. $\mathbb{Q}$, resp. $\mathbb{Z}$ ). The set of all natural numbers is denoted $\mathbb{N}: \mathbb{N}:=\{1,2, \ldots\}$; set $\mathbb{N}_{0}:=\mathbb{N} \cup\{0\}$. We use the usual notation $u \vee v:=\max (u, v), u \wedge v:=\min (u, v)$, and $u^{+}:=u \vee 0$. If $s<t$, we will use the notation $s \vee u \wedge t$ to mean $(s \vee u) \wedge t=$ $s \vee(u \wedge t)$. The expression inf $\emptyset$ is always interpreted as $\infty$.

The set of all càdlàg (i.e., continuous on the right and having limits on the left) functions $\omega:[0, T] \rightarrow \mathbb{R}$, where $T>0$, will be denoted $D[0, T]$. If $\omega \in$ $D[0, T]$ and $t \in(0, T]$, we set $\omega(t-):=\lim _{s \uparrow t} \omega(s)$ and $\Delta \omega(t):=\omega(t)-\omega(t-)$. As usual, $C^{n}(\mathbb{R})$ stands for the set of all functions $f: \mathbb{R} \rightarrow \mathbb{R}$ that are $n$ times continuously differentiable.

\section{Pathwise quadratic variation}

Let $\omega:[0, T] \rightarrow \mathbb{R}$ be a càdlàg function, interpreted as the price path of a financial security over the time period $[0, T]$ whose end-point $T>0$ is fixed throughout the paper. In this section we give a modification of Föllmer's [6] definition of the quadratic variation of $\omega$; Föllmer's definition itself will be discussed in Section 6 .

A partition (of $[0, T]$ ) is a finite sequence of numbers $0=t_{0}<t_{1}<\cdots<$ $t_{m} \leq T$; we also set $t_{k}:=\infty$ for $k>m$. The mesh of this partition is $\max _{k \in \mathbb{N}}\left|\left(t_{k} \wedge T\right)-\left(t_{k-1} \wedge T\right)\right|$.

Let $\pi=\left(\pi^{0}, \pi^{1}, \pi^{2}, \ldots\right)$ be a nested sequence of partitions: for each $n \in \mathbb{N}$, each element of $\pi^{n-1}$ is an element of $\pi^{n}$ (in this paper we concentrate on nested sequences of partitions). The $n$th approximation, $n=0,1,2, \ldots$, to the quadratic variation of $\omega$ along $\pi$ is defined by

$$
A_{t}^{n, \pi}:=\sum_{k=1}^{\infty}\left(\omega\left(\pi_{k}^{n} \wedge t\right)-\omega\left(\pi_{k-1}^{n} \wedge t\right)\right)^{2}, \quad t \in[0, T],
$$

where, for each $n \in \mathbb{N}_{0}, \pi_{k}^{n}, k=0,1, \ldots$, are the elements of $\pi^{n}: \pi^{n}=$ $\left(\pi_{0}^{n}, \pi_{1}^{n}, \pi_{2}^{n}, \ldots\right)$ and there is $m=m(n) \in \mathbb{N}_{0}$ such that $0=\pi_{0}^{n}<\pi_{1}^{n}<\cdots<$ $\pi_{m}^{n} \leq T$ and $\pi_{k}^{n}=\infty$ for all $k>m$. By $A^{n, \pi}$ we will mean the function $t \in[0, T] \mapsto A_{t}^{n, \pi}$ in $D[0, T]$.

In this paper we will be interested in the uniform metric on $D[0, T]$ :

$$
\rho\left(\omega, \omega^{\prime}\right):=\sup _{t \in[0, T]}\left|\omega(t)-\omega^{\prime}(t)\right|
$$

(This is an unusual metric for $D[0, T]$, but standard for the set $C[0, T]$ of continuous functions on $[0, T]$.) Notice that $D[0, T]$ is complete in the metric $\rho$; this will be used in the proof of Theorem 1 below.

We say that $\omega$ has quadratic variation along $\pi$, where $\pi$ is a nested sequence of partitions, if the sequence $A^{n, \pi}$ converges in the uniform metric $\rho$. The limit, when it exists, is denoted $A^{\pi}$ and called the quadratic variation of $\omega$ along $\pi$. 
A sequence $\pi$ of partitions is dense if $\lim _{n \rightarrow \infty} \operatorname{mesh}\left(\pi^{n}\right)=0$, where $\operatorname{mesh}\left(\pi^{n}\right)$ is the mesh of $\pi^{n}$. Quadratic variation along a sequence of partitions is usually (see, e.g., 6, 14]) defined only for dense sequences of partitions. The sequences of partitions considered in this paper are not always dense, and instead we will use the following property. For $\omega \in D[0, T]$, we say that a nested sequence $\pi$ of partitions $\pi^{n}, n=0,1,2, \ldots$, exhausts $\omega$ if:

- each $t \in(0, T]$ such that $\Delta \omega(t) \neq 0$ belongs to $\pi^{n}$ for some $n$ (equivalently, from some $n$ on);

- each open interval $(u, v)$ in which $\omega$ is not constant contains at least one element of $\pi^{n}$ for some $n$ (equivalently, from some $n$ on).

For $\omega \in D[0, T]$ and an interval $I \subseteq[0, T]$ set $\mathrm{w}_{\omega} I:=\sup _{s_{1}, s_{2} \in I} \mid \omega\left(s_{2}\right)-$ $\omega\left(s_{1}\right) \mid$. The oscillation of $\omega \in D[0, T]$ over a partition $\pi^{n}$ is defined as

$$
\operatorname{osc}_{\pi^{n}}(\omega):=\max _{k \in \mathbb{N}} \mathrm{w}_{\omega}\left[\pi_{k-1}^{n} \wedge T, \pi_{k}^{n} \wedge T\right)=\max _{k \in \mathbb{N}} \mathrm{w}_{\omega}\left(\pi_{k-1}^{n} \wedge T, \pi_{k}^{n} \wedge T\right) .
$$

Lemma 1. If a nested sequence of partitions $\pi$ exhausts $\omega \in D[0, T]$,

$$
\lim _{n \rightarrow \infty} \operatorname{osc}_{\pi^{n}}(\omega)=0
$$

Proof. Let $\epsilon>0$. There exist points $0=t_{0}<t_{1}<\cdots<t_{r}=T$ such that $\mathrm{w}_{\omega}\left[t_{i-1}, t_{i}\right)<\epsilon$ for all $i \in\{1, \ldots, r\}$ ([1, Lemma 14.1). Let $n$ be so large that $\pi^{n}$ contains all $t_{i}, i \in\{1, \ldots, r\}$, with $\Delta \omega\left(t_{i}\right) \neq 0$ and contains a point in each interval $\left(t_{i-1}, t_{i}\right), i \in\{1, \ldots, r\}$, such that $\omega$ is not constant in $\left(t_{i-1}, t_{i}\right)$. For any $k \in \mathbb{N}_{0}$, the interval $\left(\pi_{k}^{n} \wedge T, \pi_{k+1}^{n} \wedge T\right)$ does not contain any points $t_{i}$ with $\Delta \omega\left(t_{i}\right) \neq 0$ and does not contain any intervals $\left(t_{i-1}, t_{i}\right), i \in\{1, \ldots, r\}$, where $\omega$ is not constant. Therefore, $\mathrm{w}_{\omega}\left(\pi_{k}^{n} \wedge T, \pi_{k+1}^{n} \wedge T\right)<2 \epsilon$. Since $\epsilon$ can be arbitrarily small, this completes the proof of 2.2 . .

Lemma 2. Suppose $\omega \in D[0, T]$ and $\pi$ is a nested sequence of partitions that exhausts $\omega$. If the quadratic variation $A^{\pi}$ of $\omega$ along $\pi$ exists, it is a nondecreasing càdlàg function satisfying $A_{0}^{\pi}=0$ with jumps $\Delta A_{t}^{\pi}=(\Delta \omega(t))^{2}$ for all $t \in(0, T]$.

Proof. The equality $A_{0}^{\pi}=0$ is obvious, and $A^{\pi}$ is càdlàg as the uniform limit of càdlàg functions. (These statements do not rely on $\pi$ exhausting $\omega$, but the other statements of the lemma do require a density condition for $\pi$ : they fail, for example, when $\pi^{0}=\pi^{1}=\cdots=(0, \infty, \infty, \ldots)$.)

Let us now prove that $A^{\pi}$ is nondecreasing. If it is not, there exist $t_{1}<t_{2} \leq$ $T$ and $\epsilon \in(0,1)$ such that $A_{t_{1}}^{\pi}>A_{t_{2}}^{\pi}+2 \epsilon$. For sufficiently large $n$, we will have

$$
A_{t_{1}}^{n, \pi}>A_{t_{2}}^{n, \pi}+\epsilon
$$

Let $n$ be so large that, in addition, $\operatorname{osc}_{\pi^{n}}(\omega)<\epsilon$ (cf. Lemma 1). Let $t_{1} \in$ $\left[\pi_{k^{\prime}-1}^{n}, \pi_{k^{\prime}}^{n}\right)$ and $t_{2} \in\left[\pi_{k^{\prime \prime}}^{n}, \pi_{k^{\prime \prime}+1}^{n}\right)$; notice that $k^{\prime \prime} \geq k^{\prime}$. Since $A_{t_{2}}^{n, \pi} \geq A_{\pi_{k^{\prime \prime}}}^{n, \pi}$ 
and $A_{\pi_{k+1}^{n}}^{n, \pi} \geq A_{\pi_{k}^{n}}^{n, \pi}$ for $k=k^{\prime}, \ldots, k^{\prime \prime}-1,2.3$ implies $A_{t_{1}}^{n, \pi}>A_{\pi_{k^{\prime}}^{n}}^{n, \pi}+\epsilon$. This, however, contradicts

$$
\begin{aligned}
A_{t_{1}}^{n, \pi}-A_{\pi_{k^{\prime}}^{n}}^{n, \pi}=\left(\omega\left(t_{1}\right)-\omega\left(\pi_{k^{\prime}-1}^{n}\right)\right)^{2}-\left(\omega\left(\pi_{k^{\prime}}^{n}\right)\right. & \left.-\omega\left(\pi_{k^{\prime}-1}^{n}\right)\right)^{2} \\
& \leq\left(\omega\left(t_{1}\right)-\omega\left(\pi_{k^{\prime}-1}^{n}\right)\right)^{2}<\epsilon^{2} .
\end{aligned}
$$

It remains to prove that $\Delta A_{t}^{\pi}=(\Delta \omega(t))^{2}$ for all $t \in(0, T]$. Fix $t \in(0, T]$ and $\epsilon>0$. Let $n$ be so large that $\operatorname{osc}_{\pi^{n}}(\omega)<\epsilon$ (cf. Lemma 1). Define $k \in \mathbb{N}_{0}$ by the condition $t \in\left(\pi_{k}^{n}, \pi_{k+1}^{n}\right]$. Since

$$
\begin{aligned}
\Delta A_{t}^{n, \pi}=\left(\omega(t)-\omega\left(\pi_{k}^{n}\right)\right)^{2}-(\omega(t-) & \left.-\omega\left(\pi_{k}^{n}\right)\right)^{2} \\
& =(\Delta \omega(t))^{2}+2 \Delta \omega(t)\left(\omega(t-)-\omega\left(\pi_{k}^{n}\right)\right),
\end{aligned}
$$

we have $\left|\Delta A_{t}^{n, \pi}-(\Delta \omega(t))^{2}\right| \leq 2 \epsilon|\Delta \omega(t)|$. As $\Delta A_{t}^{n, \pi} \rightarrow \Delta A_{t}^{\pi}$ and $\epsilon$ can be arbitrarily small, we obtain $\Delta A_{t}^{\pi}=(\Delta \omega(t))^{2}$.

\section{$3 \quad$ Typical price paths}

We consider a perfect-information game between two players called Reality (financial market) and Sceptic (speculator). Reality outputs a càdlàg function $\omega:[0, T] \rightarrow \mathbb{R}$, interpreted as the price path of a financial security, and Sceptic tries to profit by trading in $\omega$. First Sceptic presents his trading strategy and then Reality chooses $\omega$. In the first two subsections of this section we will formalize this picture (often following [22]) by defining the allowed moves for Reality and strategies for Sceptic.

\section{Sample space}

Let $\psi:[0, \infty) \rightarrow(0, \infty)$ be a nondecreasing function, fixed through most of this paper. The set of allowed moves for Reality (our sample space) is

$$
\Omega_{\psi}:=\left\{\omega \in D[0, T]|\forall t \in(0, T]:| \Delta \omega(t) \mid \leq \psi\left(\sup _{s \in[0, t)}|\omega(s)|\right)\right\} .
$$

The function $\psi$ determines the allowed size of the jumps. It can be arbitrarily large (but needs, however, to be known in advance). Our conclusions (e.g., in Theorems 1 and 2 will not depend on $\psi$; therefore, our results become stronger as $\psi$ becomes larger.

Two natural examples of restrictions on the jumps are:

- The absolute values $|\Delta \omega(t)|$ of $\omega$ 's jumps never exceed a known constant $c>0$. Such price paths $\omega$ belong to $\Omega_{c}$.

- The price path $\omega$ is known to be nonnegative, and the relative values $\Delta \omega(t) / \sup _{s \in[0, t)}|\omega(s)|$ (with $0 / 0:=0$ ) of $\omega$ 's jumps w.r. to their largest 
value so far never exceed a known constant $c>0$. Such $\omega$ belong to $\Omega_{\psi}$, where $\psi(u)=(c \vee 1) u$. (There is no need to explicitly restrict downward jumps when $\omega \geq 0$ : they are restricted automatically by the current value of the security.)

The first example is the simplest one mathematically and will be used in the proof of our main result in Section 4. The second example is more relevant to many real financial markets, and its generalized and elaborated version will be discussed at the end of this section.

\section{Trading strategies}

For each $t \in[0, T], \mathcal{F}_{t}^{\circ}$ is defined to be the smallest $\sigma$-algebra on $\Omega_{\psi}$ that makes all functions $\omega \mapsto \omega(s), s \in[0, t]$, measurable; $\mathcal{F}_{t}$ is defined as the universal completion of $\mathcal{F}_{t}^{\circ}$. A process (more fully, adapted process) $S$ is a family of functions $S_{t}: \Omega_{\psi} \rightarrow[-\infty, \infty], t \in[0, T]$, each $S_{t}$ being $\mathcal{F}_{t}$-measurable. An event is an element of the $\sigma$-algebra $\mathcal{F}_{T}$. Stopping times $\tau: \Omega_{\psi} \rightarrow[0, T] \cup\{\infty\}$ w.r. to the filtration $\left(\mathcal{F}_{t}\right)$ and the corresponding $\sigma$-algebras $\mathcal{F}_{\tau}$ are defined as usual; $\omega(\tau(\omega))$ and $S_{\tau(\omega)}(\omega)$ will often be simplified to $\omega(\tau)$ and $S_{\tau}(\omega)$, respectively.

The class of allowed strategies for Sceptic is defined in two steps. A simple trading strategy $G$ consists of: (a) a nondecreasing infinite sequence of stopping times $\tau_{1} \leq \tau_{2} \leq \cdots$ such that, for each $\omega \in \Omega_{\psi}, \tau_{n}(\omega)<\infty$ for only finitely many $n$; (b) for each $n=1,2, \ldots$, a bounded $\mathcal{F}_{\tau_{n}}$-measurable function $h_{n}$. To such $G$ and an initial capital $\alpha \in \mathbb{R}$ corresponds the simple capital process

$$
\mathcal{K}_{t}^{G, \alpha}(\omega):=\alpha+\sum_{n=1}^{\infty} h_{n}(\omega)\left(\omega\left(\tau_{n+1} \wedge t\right)-\omega\left(\tau_{n} \wedge t\right)\right), \quad t \in[0, T]
$$

the value $h_{n}(\omega)$ will be called the position taken at time $\tau_{n}$, and $\mathcal{K}_{t}^{G, \alpha}(\omega)$ will sometimes be referred to as Sceptic's capital at time $t$. Notice that the sum of finitely many simple capital processes is again a simple capital process.

A nonnegative capital process is any process $S$ that can be represented in the form

$$
S_{t}(\omega):=\sum_{n=1}^{\infty} \mathcal{K}_{t}^{G_{n}, \alpha_{n}}(\omega),
$$

where the simple capital processes $\mathcal{K}_{t}^{G_{n}, \alpha_{n}}(\omega)$ are required to be nonnegative, for all $t$ and $\omega$, and the nonnegative series $\sum_{n=1}^{\infty} \alpha_{n}$ is required to converge in $\mathbb{R}$ (intuitively, the total capital invested has to be finite). The sum (3.3) is

always nonnegative, but we allow it to take value $+\infty$. Since $\mathcal{K}_{0}^{G_{n}, \alpha_{n}}(\omega)=\alpha_{n}$ does not depend on $\omega, S_{0}(\omega)$ also does not depend on $\omega$ and will sometimes be abbreviated to $S_{0}$.

\section{Upper price}

The upper price of a set $E \subseteq \Omega_{\psi}$ is defined as

$$
\overline{\mathbb{P}}(E):=\inf \left\{S_{0} \mid \forall \omega \in \Omega_{\psi}: S_{T}(\omega) \geq \mathbf{1}_{E}(\omega)\right\},
$$


where $S$ ranges over the nonnegative capital processes and $\mathbf{1}_{E}$ stands for the indicator of $E$. Notice that $\overline{\mathbb{P}}\left(\Omega_{\psi}\right)=1$ (in the terminology of [17, our game protocol is "coherent"): indeed, $\overline{\mathbb{P}}\left(\Omega_{\psi}\right)<1$ would mean that some nonnegative capital process increases between time 0 and $T$ for all $\omega \in \Omega_{\psi}$, and this is clearly impossible for constant $\omega$.

Remark 1. The notion of upper price (introduced in 20]) is similar to the notion of upper hedging price (see, e.g., 7], Section 7.3) except that the latter replaces the requirement $\forall \omega: S_{T}(\omega) \geq \mathbf{1}_{E}(\omega)$ in (3.4) by " $S_{T} \geq \mathbf{1}_{E}$ a.s.", thus requiring a statistical model for prices (which is, however, only used via its family of events of probability zero).

Remark 2. An alternative probability-free definition of upper price is given in [15, Definition 1, and [16], Definition 3.1. See [15], Section 2.1, for a comparison of the two definitions; an advantage of our definition for the purpose of this paper is that our mathematical results are at least as strong (and possibly stronger) when they use our definition than when they use the definition of [15, 16] (see [16], Lemma 10).

We say that $E \subseteq \Omega_{\psi}$ is null if $\overline{\mathbb{P}}(E)=0$. A property of $\omega \in \Omega_{\psi}$ will be said to hold for typical $\omega$ if the set of $\omega$ where it fails is null. Correspondingly, a set $E \subseteq \Omega_{\psi}$ is almost certain if $\overline{\mathbb{P}}\left(\Omega_{\psi} \backslash E\right)=0$. All null sets $E$ are automatically strictly null: there exists a nonnegative capital process $S$ with $S_{0}=1$ such that $S_{T}(\omega)=\infty$ for all $\omega \in E$ (indeed, if $E$ is null, we can sum nonnegative capital processes $S^{n}, n \in \mathbb{N}$, such that $S_{0}^{n}=2^{-n}$ and $S_{T}^{n} \geq \mathbf{1}_{E}$ ). Notice that the union of countably (in particular, finitely) many null sets is also a null set.

\section{Our definitions in view of margin requirements}

In the rest of this section we discuss our definitions in view of the margin requirements that traders usually have to comply with. Suppose, for concreteness, that $\omega$ is the price path of a common stock. We then have $\omega(t) \geq 0$ for all $t \in[0, T]$. Our definition (3.2) implicitly assumes the following picture. Sceptic starts from the amount $\alpha$ in his margin account, and he never adds funds to or withdraws funds from the account. He is allowed to take both positive and negative positions in the stock (can go both long and short), but the capital in his account should always stay nonnegative. Let us consider a somewhat more realistic picture where Sceptic is required not only to keep the capital in the margin account nonnegative but also to satisfy margin requirements.

In general, different margin requirements apply to long and short positions. The rules can be summarized as follows. In the case of a long position, the capital in the margin account should not only be nonnegative but should stay nonnegative in the imaginary event that the stock price immediately drops by $100 c_{\mathrm{lg}} \%$, where $c_{\mathrm{lg}}$ is the minimum margin requirement for long positions. In the case of a short position, the capital in the margin account should not only be nonnegative but should stay nonnegative in the imaginary event that the stock price immediately rises by $100 c_{\mathrm{sh}} \%$, where $c_{\mathrm{sh}}$ is the minimum margin 
requirement for short positions. Different values may be used for $c_{\mathrm{lg}}$ and $c_{\mathrm{sh}}$ at the time when the position is opened and at later times; they are called the initial and maintenance margin requirements, respectively. The current initial margin requirements stipulated by the Federal Reserve Board [5] are the same for both long and short positions: $c_{\mathrm{lg}}=c_{\mathrm{sh}}=0.5$. The maintenance margin requirement never exceeds the initial margin requirement, and we make Sceptic's task harder by setting the former to the latter. As $\omega \geq 0$, we always assume $c_{\lg } \leq 1$.

If the margin requirement becomes violated, the trader receives a request, known as a margin call, to add funds to the account. If the margin call is ignored, the account can be liquidated. We will assume that the margin account provides "non-recourse" loans on the part of the broker, so that the trader is not responsible for any possible shortfall after liquidation.

Remark 3. Although being non-recourse is a common feature of some related kinds of loan, such as stock loans, margin loans themselves are legally recourse loans in the USA. However, the assumption that margin loans are non-recourse is not unusual $([8$, p. 29) and there is a view that "margin loans, while legally recourse loans, might be in a limbo, somewhere between recourse and nonrecourse" ([8], p. 38).

The trading strategy developed in this paper (see Theorem 1) starts with one monetary unit, makes sure that the capital is always nonnegative, and brings infinite capital if the quadratic variation of $\omega$ does not exist; it, however, assumes that $\omega \in \Omega_{\psi}$. (What it means for the quadratic variation of $\omega \in \Omega_{\psi}$ to exist will be defined in the next section and is not important for the current discussion.) The strategy, however, has two disadvantages:

- whereas ensuring that its capital is always nonnegative when $\omega \in \Omega_{\psi}$, it is not guaranteed to satisfy the margin requirements;

- the strategy can lead to a negative capital when $\omega \notin \Omega_{\psi}$.

If we, however, apply our result to the sample space $\Omega_{\psi^{\prime}}$ in place of $\Omega_{\psi}$, where

$$
\psi^{\prime}(u):=1 \vee\left(\left(1+c_{\mathrm{sh}}\right) \psi(u)+c_{\mathrm{sh}} u\right),
$$

we will obtain a trading strategy satisfying the following stronger guarantees: it still starts with one monetary unit; it makes sure that the margin requirements are satisfied if $\omega \in \Omega_{\psi}$; it brings infinite capital if $\omega \in \Omega_{\psi}$ but the quadratic variation of $\omega$ does not exist; it never loses more than the one monetary unit (because the strategy always ignores margin calls).

Let us check that the trading strategy constructed for $\Omega_{\psi^{\prime}}$ will indeed satisfy the margin requirements for each of the constituent accounts (corresponding to the addends in (3.3)), supposing $\omega \in \Omega_{\psi}$. Since $\psi^{\prime} \geq 1$, at each time when the position is long the capital in the account will be no less than the value of the stock in the account at this time (otherwise, a 100\% downward jump in the price of $\omega$ would have led to a negative capital). Since $c_{\mathrm{lg}} \leq 1$, the margin 
requirement will be satisfied. It remains to consider a time $t$ when the position is short. The worst case is when the price jumps up by the largest allowed amount becoming $\omega(t)+\psi\left(\sup _{s \in[0, t)} \omega(s)\right)$. The margin requirements will be satisfied if the capital resulting from the imaginary event that the price again jumps up by a factor of $1+c_{\mathrm{sh}}$ is still nonnegative. This is guaranteed by our strategy since, by 3.5 ,

$$
\left(1+c_{\mathrm{sh}}\right)\left(\omega(t)+\psi\left(\sup _{s \in[0, t)} \omega(s)\right)\right) \leq \omega(t)+\psi^{\prime}\left(\sup _{s \in[0, t)} \omega(s)\right) .
$$

\section{Existence of quadratic variation}

In this section we define a suitable sequence of partitions $\tau^{n}(\omega)$ for each $\omega \in$ $\Omega_{\psi}$ and show that the quadratic variation of $\omega$ along this sequence exists for typical $\omega$.

For each $n \in \mathbb{N}_{0}$, let $\mathbb{D}^{n}:=\left\{k 2^{-n} \mid k \in \mathbb{Z}\right\}$ and define a sequence of stopping times $\tau_{k}^{n}, k=0,1,2, \ldots$, and a sequence $D_{k}^{n}, k=0,1,2, \ldots$, of $\mathcal{F}_{\tau_{k}^{n}}$-measurable functions inductively by $\tau_{0}^{n}:=0, D_{0}^{n}:=\sup \left(\mathbb{D}^{n} \cap(-\infty, \omega(0)]\right)$,

$$
\begin{gathered}
\tau_{k}^{n}(\omega):=\inf \left\{t \in\left[\tau_{k-1}^{n}(\omega), T\right] \mid \llbracket \omega\left(\tau_{k-1}^{n}\right), \omega(t) \rrbracket \cap\left(\mathbb{D}^{n} \backslash\left\{D_{k-1}^{n}(\omega)\right\}\right) \neq \emptyset\right\}, \\
D_{k}^{n}(\omega) \in \operatorname{argmin}_{D \in \llbracket \omega\left(\tau_{k-1}^{n}\right), \omega\left(\tau_{k}^{n}\right) \rrbracket \cap\left(\mathbb{D}^{n} \backslash\left\{D_{k-1}^{n}(\omega)\right\}\right)}\left|D-\omega\left(\tau_{k}^{n}\right)\right|
\end{gathered}
$$

for $k=1,2, \ldots$, where we use the notation

$$
\llbracket u, v \rrbracket:= \begin{cases}{[u, v]} & \text { if } u \leq v \\ {[v, u]} & \text { if } u>v\end{cases}
$$

for the convex closure of the set $\{u, v\}$. Notice that the argmin in 4.1) is a one-element set, and so $D_{k}^{n}(\omega)$ is determined uniquely.

We will check that $\tau_{k}^{n}$ are indeed stopping times and that $D_{k}^{n}$ are $\mathcal{F}_{\tau_{k}^{n-}}$ measurable in Lemma 3 below. If $\tau_{k}^{n}(\omega)=\infty$ (arising from our convention $\inf \emptyset:=\infty)$, we set, e.g., $D_{k}^{n}(\omega):=0$. The intuition behind $D_{k}^{n}$ is that it is the current element of $\mathbb{D}^{n}$ at time $\tau_{k}^{n}$; when $\omega$ is continuous, $D_{k}^{n}=\omega\left(\tau_{k}^{n}\right)$ (this is the case considered in [21]), but in general we only have $\left|D_{k}^{n}-\omega\left(\tau_{k}^{n}\right)\right|<2^{-n}$, assuming $\tau_{k}^{n}<\infty$. Let $\tau^{n}(\omega)$ be the partition $0=\tau_{0}^{n}(\omega) \leq \tau_{1}^{n}(\omega) \leq \tau_{2}^{n}(\omega) \leq \cdots$ (in fact, we will have $\tau_{k}^{n}(\omega)<\tau_{k+1}^{n}(\omega)$ unless $\tau_{k}^{n}(\omega)=\infty$ ), and let $\tau(\omega)$ be the sequence of the partitions $\tau^{n}(\omega)$.

Theorem 1. Let $\psi:[0, \infty) \rightarrow(0, \infty)$ be a nondecreasing function. Typical $\omega \in \Omega_{\psi}$ have quadratic variation along $\tau(\omega)$.

The theorem says that for typical $\omega \in \Omega_{\psi}$ the sequence of functions

$$
A_{t}^{n}(\omega):=\sum_{k=1}^{\infty}\left(\omega\left(\tau_{k}^{n} \wedge t\right)-\omega\left(\tau_{k-1}^{n} \wedge t\right)\right)^{2}, \quad n=0,1,2, \ldots,
$$


converges in the uniform metric to a function $A(\omega) \in D[0, T]$. (In this section, we omit mentioning the sequence of partitions in our notation for quadratic variation.) Since the sequence of partitions $\tau^{n}(\omega)$ is always nested and exhausts $\omega$ (see Lemma 3 below), by Lemma 2 the limit $A(\omega)$ will be a nondecreasing function satisfying $A_{0}(\omega)=0$ and $\Delta A_{t}(\omega)=(\Delta \omega(t))^{2}$ for all $t \in(0, T]$.

The following lemma lists several useful properties of $\tau_{k}^{n}$ and $D_{k}^{n}$.

Lemma 3. The functions $\tau_{k}^{n}: \Omega_{\psi} \rightarrow[0, \infty]$ and $D_{k}^{n}: \Omega_{\psi} \rightarrow \mathbb{R}$ satisfy the following properties:

(a) the infimum in the definition of $\tau_{k}^{n}(\omega)$ is attained, provided $\tau_{k}^{n}(\omega)<\infty$;

(b) $\tau_{k}^{n}>\tau_{k-1}^{n}$ unless $\tau_{k-1}^{n}=\infty$;

(c) for each $n, \tau_{k}^{n}<\infty$ for only finitely many $k$;

(d) the sequences $\tau^{n}$ are nested: for all $n, k \in \mathbb{N}_{0}$ there is $k^{\prime}$ such that $\tau_{k^{\prime}}^{n+1}=$ $\tau_{k}^{n}$;

(e) for each $\omega \in \Omega_{\psi}$, the sequence of partitions $\tau^{n}(\omega)$ exhausts $\omega$;

(f) $\tau_{k}^{n}$ are bona fide stopping times and $D_{k}^{n}$ are $\mathcal{F}_{\tau_{k}^{n}}$-measurable.

Proof. Parts (a) and (b) follow from the right-continuity of $\omega$.

Part (c) follows from the observation that the total number of upcrossings and downcrossings of the intervals of the form $\left(i 2^{-n},(i+1) 2^{-n}\right), i \in \mathbb{Z}$, by $\omega$ over the time interval $\left[0, \tau_{k}^{n}\right]$ is at least $k-1$; remember that functions in $D[0, T]$ are bounded and can cross any non-empty interval only finitely often (4), Theorem 4.22).

Part (d) can be easily proved by induction in $k$ if it is strengthened as follows: for each $n \in \mathbb{N}_{0}$ there is a (unique) sequence $0=i_{0} \leq i_{1} \leq \cdots$ such that $\tau_{i_{k}}^{n+1}=\tau_{k}^{n}$ and $\left|D_{i_{k}}^{n+1}-D_{k}^{n}\right| \leq 2^{-n-1}$.

Part (e) is obvious.

It remains to prove part (f) Fix $n \in \mathbb{N}_{0}$; we will use induction in $k\left(\tau_{0}^{n}=0\right.$ is obviously a stopping time). Fix $k \in \mathbb{N}$ and suppose that $\tau_{k-1}^{n}$ is a stopping time and $D_{k-1}^{n}$ is $\mathcal{F}_{\tau_{k-1}^{n}}$-measurable. Let $t \in(0, T]$. The condition $\tau_{k}^{n}(\omega) \leq t$ on $\omega$ can be rewritten as:

$$
\begin{aligned}
\exists u \in(0, t) \cap \mathbb{Q} \exists D \in \mathbb{D}^{n}: & \tau_{k-1}^{n} \leq u \& D_{k-1}^{n}=D \& \\
& \exists s \in(u, t]: \llbracket \omega(u), \omega(s) \rrbracket \cap\left(\mathbb{D}^{n} \backslash\{D\}\right) \neq \emptyset
\end{aligned}
$$

(this uses the right-continuity of $\omega$ ). To complete the proof that $\tau_{k}^{n}$ is a stopping time, it suffices to prove that for any rational number $u \in(0, t)$ and any $D \in \mathbb{D}^{n}$, the event (4.4) belongs to $\mathcal{F}_{t}$.

It is clear that (4.4) can be represented as the union of events of the form

$$
\begin{aligned}
\left\{\exists s \in(u, t]: \omega(u) \leq D^{\prime} \& \omega(s)\right. & \left.\geq D^{\prime \prime}\right\} \\
& =\left\{\omega(u) \leq D^{\prime}\right\} \cap\left\{\exists s \in(u, t]: \omega(s) \geq D^{\prime \prime}\right\}
\end{aligned}
$$


(perhaps with " $\leq$ " and " $\geq$ " interchanged) for some $D^{\prime}, D^{\prime \prime} \in \mathbb{D}^{n}$. Therefore, it suffices to prove that the event

$$
\exists s \in(u, t]: \omega(s) \geq D^{\prime \prime}
$$

is in $\mathcal{F}_{t}$ (the case with " $\leq$ " in place of " $\geq$ " is treated analogously).

The event (4.5) is the projection onto $\Omega_{\psi}$ of the set $A:=\{(s, \omega) \in(u, t] \times$ $\left.\Omega_{\psi} \mid \omega(s) \geq D^{\prime \prime}\right\}$. Since càdlàg processes are progressively measurable, $A \in$ $\mathcal{B}_{t} \times \mathcal{F}_{t}^{\circ}$, where $\mathcal{B}_{t}$ is the Borel $\sigma$-algebra on $[0, t]$ and $\mathcal{B}_{t} \times \mathcal{F}_{t}^{\circ}$ is the product $\sigma$ algebra. This implies that the projection 4.5 is an $\mathcal{F}_{t}^{\circ}$-analytic set (according to [4], Theorem III.13(3)), which in turn implies that this set is in the universal completion $\mathcal{F}_{t}$ of $\mathcal{F}_{t}^{\circ}$ (according to [4], Theorem III.33).

We can see that $\tau_{k}^{n}$ is a stopping time. By induction in $k$, each $D_{k}^{n}$ is $\tau_{k}^{n}$ measurable: indeed, for $D \in \mathbb{D}^{n}, D_{k}^{n}=D$ is equivalent to $\omega\left(\tau_{k}^{n}\right) \in\left[D, D+2^{-n}\right)$ when $D>D_{k-1}^{n}$, is equivalent to $\omega\left(\tau_{k}^{n}\right) \in\left(D-2^{-n}, D\right]$ when $D<D_{k-1}^{n}$, and is impossible when $D=D_{k-1}^{n}$.

\section{Auxiliary results}

The rest of this section is devoted to the proof of Theorem 1. First we notice that it suffices to prove Theorem 1 only in the case where $\psi$ is constant, $\psi=c$ for $c>0$. Indeed, suppose Theorem 1 holds for all $\psi=c$ and let us prove it for general nondecreasing $\psi$. Since functions in $D[0, T]$ are bounded and unions of countably many null sets are null, it suffices to prove, for a fixed $L \in \mathbb{N}$, that typical $\omega \in \Omega_{\psi}$ satisfying $\sup _{t \in[0, T]}|\omega(t)| \leq 2^{L}$ have quadratic variation along $\tau(\omega)$. The latter can be achieved by: (a) running a trading strategy risking at most one monetary unit and bringing infinite capital when $\omega \in \Omega_{c}$ for $c:=\psi\left(2^{L}\right)$ and $\omega$ does not have quadratic variation along $\tau(\omega)$, and (b) stopping trading at the time

$$
\sigma_{L}(\omega):=\inf \left\{t \in[0, T]|| \omega(t) \mid \geq 2^{L}\right\}
$$

(this is a stopping time: see the proof of Lemma $3(\mathrm{f})$.).

Next we prove several auxiliary lemmas, assuming $\psi=c$ for $c \geq 1$ (there is no loss of generality in the further assumption $c \geq 1$ ).

Lemma 4. For each $n \in \mathbb{N}$, the process $S_{t}^{n}:=A_{t}^{n}-A_{t}^{n-1}$ is a simple capital process.

Proof. We will show that $S_{t}^{n}$ is the capital process of a simple trading strategy that changes its position only at times $\tau_{k}^{n}$. We have, for $t \in\left[\tau_{k}^{n}, \tau_{k+1}^{n}\right] \cap[0, T]$ :

$$
\begin{aligned}
S_{t}^{n}-S_{\tau_{k}^{n}}^{n} & =\left(A_{t}^{n}-A_{t}^{n-1}\right)-\left(A_{\tau_{k}^{n}}^{n}-A_{\tau_{k}^{n}}^{n-1}\right) \\
& =\left(A_{t}^{n}-A_{\tau_{k}^{n}}^{n}\right)-\left(A_{t}^{n-1}-A_{\tau_{k}^{n}}^{n-1}\right) \\
& =\left(\omega(t)-\omega\left(\tau_{k}^{n}\right)\right)^{2}-\left((\omega(t)-\omega(\tau))^{2}-\left(\omega\left(\tau_{k}^{n}\right)-\omega(\tau)\right)^{2}\right) \\
& =-2\left(\omega\left(\tau_{k}^{n}\right)-\omega(\tau)\right)\left(\omega(t)-\omega\left(\tau_{k}^{n}\right)\right),
\end{aligned}
$$


where $\tau:=\max \left\{\tau_{k^{\prime}}^{n-1} \mid \tau_{k^{\prime}}^{n-1} \leq \tau_{k}^{n}\right\}$. Therefore, it suffices to take position $-2\left(\omega\left(\tau_{k}^{n}\right)-\omega(\tau)\right)$ at time $\tau_{k}^{n}$.

The proof of Theorem 1 involves the following simple capital process (based on a standard idea going back to at least Kolmogorov [9]):

$$
U_{t}^{n}:=2^{-2 n+8} c^{2}+n^{4} 2^{-2 n}+\left(S_{t}^{n}\right)^{2}-\sum_{k=1}^{\infty}\left(S_{\tau_{k}^{n} \wedge t}^{n}-S_{\tau_{k-1}^{n} \wedge t}^{n}\right)^{2} .
$$

Lemma 5. For each $n \in \mathbb{N}, U_{t}^{n}$ is indeed a simple capital process.

Proof. As in the previous lemma, the position is only changed at times $\tau_{k}^{n}$. When $t \in\left[\tau_{k}^{n}, \tau_{k+1}^{n}\right] \cap[0, T]$, we have:

$$
\begin{aligned}
U_{t}^{n}-U_{\tau_{k}^{n}}^{n}= & \left(\left(S_{t}^{n}\right)^{2}-\sum_{i=1}^{k}\left(S_{\tau_{i}^{n}}^{n}-S_{\tau_{i-1}^{n}}^{n}\right)^{2}-\left(S_{t}^{n}-S_{\tau_{k}^{n}}^{n}\right)^{2}\right) \\
& -\left(\left(S_{\tau_{k}^{n}}^{n}\right)^{2}-\sum_{i=1}^{k}\left(S_{\tau_{i}^{n}}^{n}-S_{\tau_{i-1}^{n}}^{n}\right)^{2}\right) \\
= & 2 S_{\tau_{k}^{n}}^{n}\left(S_{t}^{n}-S_{\tau_{k}^{n}}^{n}\right) \\
= & -4 S_{\tau_{k}^{n}}^{n}\left(\omega\left(\tau_{k}^{n}\right)-\omega(\tau)\right)\left(\omega(t)-\omega\left(\tau_{k}^{n}\right)\right)
\end{aligned}
$$

(the last equality follows from (4.7)). Therefore, it suffices to take position $-4 S_{\tau_{k}^{n}}^{n}\left(\omega\left(\tau_{k}^{n}\right)-\omega(\tau)\right)$ at time $\tau_{k}^{n}$.

Set

$$
\sigma^{n}:=\min \left\{\tau_{K}^{n} \mid \sum_{k=1}^{K}\left(S_{\tau_{k}^{n}}^{n}-S_{\tau_{k-1}^{n}}^{n}\right)^{2}>n^{4} 2^{-2 n}\right\} .
$$

We will be interested in the nonnegative simple capital process $U_{\sigma^{n} \wedge t}^{n}$; its nonnegativity (on $\Omega_{\psi}=\Omega_{c}$ ) follows from $\left(S_{t}^{n}-S_{\tau_{k}^{n}}^{n}\right)^{2} \leq 2^{-2 n+8} c^{2}$, where $t \in\left[\tau_{k}^{n}, \tau_{k+1}^{n}\right] \cap[0, T]$, which in turn follows from 4.7 :

$$
\begin{aligned}
\left|S_{t}^{n}-S_{\tau_{k}^{n}}^{n}\right|=2\left|\omega\left(\tau_{k}^{n}\right)-\omega(\tau)\right| \mid \omega(t) & -\omega\left(\tau_{k}^{n}\right) \mid \\
& \leq 2\left(2 \times 2^{-n+1}\right)\left(c+2 \times 2^{-n}\right) \leq 2^{-n+4} c .
\end{aligned}
$$

To analyse the process $U_{\sigma^{n} \wedge t}^{n}$ we will need a probability-free version of Doob's upcrossing inequality (Lemma 6 below) and its corollary (Lemma 7) obtained by a method proposed by Bruneau [2] and developed and simplified in [18] and 22 .

Let $\mathrm{M}_{t}^{(a, b)}(f)$ (resp. $\mathrm{D}_{t}^{(a, b)}(f)$ ) be the number of upcrossings (resp. downcrossings) of an open interval $(a, b)$ by a function $f:[0, T] \rightarrow \mathbb{R}$ during the time interval $[0, t]$. For each $h>0$ set

$$
\mathrm{M}_{t}(f, h):=\sum_{k \in \mathbb{Z}} \mathrm{M}_{t}^{(k h,(k+1) h)}(f), \quad \mathrm{D}_{t}(f, h):=\sum_{k \in \mathbb{Z}} \mathrm{D}_{t}^{(k h,(k+1) h)}(f) .
$$

Remember that $\sigma_{L}$ is the stopping time defined by 4.6 . 
Lemma 6. Let $L \in \mathbb{N}$ and $(a, b) \subseteq\left(-2^{L}, 2^{L}\right)$ be a non-empty interval. There exists a nonnegative simple capital process $V$ that starts from $V_{0}=a+2^{L}+c$ and satisfies

$$
V_{t}(\omega) \geq(b-a) \mathrm{M}_{t}^{(a, b)}(\omega)
$$

for all $\omega \in \Omega_{\psi}=\Omega_{c}$ and all $t \in\left[0, \sigma_{L}(\omega)\right]$.

(Remember that $V$ being nonnegative means that $V_{t}(\omega) \geq 0$ for all $t$ and $\omega \in$ $\left.\Omega_{\psi}.\right)$

Proof. The following standard argument will be easy to formalize. A simple trading strategy $G$ leading to $V$ can be defined as follows. The initial capital is $a+2^{L}+c$. At first $G$ takes position 0 . When $\omega$ first hits $(-\infty, a], G$ takes position 1 until $\omega$ hits $[b, \infty)$, at which point $G$ takes position 0 ; after $\omega$ hits $(-\infty, a], G$ maintains position 1 until $\omega$ hits $[b, \infty)$, at which point $G$ takes position 0 ; etc. The only exception is that trading is stopped at time $\sigma_{L}$ : the position at that time becomes 0 and stays 0 afterwards. (The essential bit is that trading should be stopped when $\omega$ hits $\left(-\infty,-2^{L}\right]$, if this ever happens.) Since $\omega$ 's jumps never exceed $c$ in absolute value, the process $S$ will be nonnegative.

Formally, we define $\tau_{1}:=\inf \{t \in[0, T] \mid \omega(t) \in(-\infty, a]\}$ and, for $n=$ $2,3, \ldots$,

$$
\tau_{n}:=\inf \left\{t \in[0, T] \mid t>\tau_{n-1} \quad \& \omega(t) \in I_{n}\right\}
$$

where $I_{n}:=[b, \infty)$ for even $n$ and $I_{n}:=(-\infty, a]$ for odd $n$. Each $\tau_{n}$ is a stopping time: this can be shown analogously to the proof of Lemma $3(\mathrm{f})$. We have $\tau_{n}(\omega)<\infty$ for only finitely many $n$ since $\omega$ is càdlàg: see, e.g., [4, Theorem IV.22.

Since $\omega$ is a right-continuous function and $(-\infty, a]$ and $[b, \infty)$ are closed sets, the infimum in the definition of $\tau_{n}, n=1,2, \ldots$, is attained when $\tau_{n}<\infty$. Therefore, $\omega\left(\tau_{1}\right) \leq a, \omega\left(\tau_{2}\right) \geq b, \omega\left(\tau_{3}\right) \leq a, \omega\left(\tau_{4}\right) \geq b$, and so on while $\tau_{n}<\infty$. Set, for $n=1,2, \ldots$,

$$
\tau_{n}^{\prime}:= \begin{cases}\tau_{n} & \text { if } \tau_{n}<\sigma_{L} \\ \sigma_{L} & \text { if } \tau_{n-1}<\sigma_{L} \leq \tau_{n} \\ \infty & \text { otherwise }\end{cases}
$$

(the inequality $\tau_{n-1}<\sigma_{L}$ is considered to be true when $n=1$ ). The position taken by $G$ at the time $\tau_{n}^{\prime}, n=1,2, \ldots$, is

$$
h_{n}:= \begin{cases}1 & \text { if } \tau_{n}^{\prime}<\sigma_{L} \text { and } n \text { is odd } \\ 0 & \text { otherwise }\end{cases}
$$

and the initial capital is $a+2^{L}+c$. Let $t \in\left[0, \sigma_{L}\right]$ and $n$ be the largest integer such that $\tau_{n} \leq t$ (with $n:=0$ when $\tau_{1}>t$; the formulas below will never involve $\left.\tau_{0}\right)$. Now we obtain from (3.2): if $n$ is even,

$$
V_{t}(\omega)=V_{0}+\left(\omega\left(\tau_{2}\right)-\omega\left(\tau_{1}\right)\right)+\left(\omega\left(\tau_{4}\right)-\omega\left(\tau_{3}\right)\right)+\cdots+\left(\omega\left(\tau_{n}\right)-\omega\left(\tau_{n-1}\right)\right)
$$




$$
\geq a+2^{L}+c+(b-a) \mathrm{M}_{t}^{(a, b)}(\omega)
$$

and if $n$ is odd,

$$
\begin{aligned}
V_{t}(\omega)= & V_{0}+\left(\omega\left(\tau_{2}\right)-\omega\left(\tau_{1}\right)\right)+\left(\omega\left(\tau_{4}\right)-\omega\left(\tau_{3}\right)\right)+\cdots+\left(\omega\left(\tau_{n-1}\right)-\omega\left(\tau_{n-2}\right)\right) \\
& +\left(\omega(t)-\omega\left(\tau_{n}\right)\right) \\
\geq & a+2^{L}+c+(b-a) \mathrm{M}_{t}^{(a, b)}(\omega)+\left(\omega(t)-\omega\left(\tau_{n}\right)\right) \\
\geq & a+2^{L}+c+(b-a) \mathrm{M}_{t}^{(a, b)}(\omega)+\left(-2^{L}-c-a\right)=(b-a) \mathrm{M}_{t}^{(a, b)}(\omega)
\end{aligned}
$$

in both cases, 4.10 holds. In particular, $V_{t}(\omega)$ is nonnegative.

Lemma 7. Let $L \in \mathbb{N}$. For each $n \in \mathbb{N}$, there exists a nonnegative simple capital process $V$ such that $V_{0} \leq 2^{L}+c$ and $V_{t}(\omega) \geq 2^{-2 n-L-1} \mathrm{M}_{t-}\left(\omega, 2^{-n}\right)$ for all $\omega \in \Omega_{c}$ and all $t \in\left[0, \sigma_{L}(\omega)\right]$.

Proof. By Lemma 6, for each $k \in\left\{-2^{L+n}, \ldots, 2^{L+n}-1\right\}$ there exists a nonnegative simple capital process $V^{k}$ that starts from $k 2^{-n}+2^{L}+c$ and satisfies

$$
V_{t}^{k}(\omega) \geq 2^{-n} \mathrm{M}_{t}^{\left(k 2^{-n},(k+1) 2^{-n}\right)}(\omega)
$$

for all $t \in\left[0, \sigma_{L}(\omega)\right]$. Summing $2^{-L-n-1} V^{k}$ over $k=-2^{L+n}, \ldots, 2^{L+n}-1$ (i.e., averaging $V^{k}$ ), we obtain a nonnegative simple capital process $V$ such that

$$
\begin{gathered}
V_{0}=2^{-L-n-1} \sum_{k=-2^{L+n}}^{2^{L+n}-1} k 2^{-n}+2^{L}+c \leq 2^{L}+c, \\
V_{t}(\omega) \geq 2^{-2 n-L-1} \mathrm{M}_{t}\left(\omega, 2^{-n}\right) \text { for all } t<\sigma_{L} ;
\end{gathered}
$$

at $t=\sigma_{L}(\omega)$ we are only guaranteed to have $V_{t}(\omega) \geq 2^{-2 n-L-1} \mathrm{M}_{t-}\left(\omega, 2^{-n}\right)$ because of the possibility $\omega(t) \notin\left[-2^{L}, 2^{L}\right]$.

Corollary 1. Let $L \in \mathbb{N}$. For typical $\omega \in \Omega_{c}$, from some $n \in \mathbb{N}$ on, $\mathrm{M}_{\sigma_{L^{-}}}\left(\omega, 2^{-n}\right) \leq n^{2} 2^{2 n}$ and $\mathrm{D}_{\sigma_{L^{-}}}\left(\omega, 2^{-n}\right) \leq n^{2} 2^{2 n}$.

Proof. It suffices to prove that $\mathrm{M}_{\sigma_{L}-}\left(\omega, 2^{-n}\right) \leq n^{1.5} 2^{2 n}$ from some $n$ on (since $\left.\mathrm{D}_{\sigma_{L}-}\left(\omega, 2^{-n}\right) \leq \mathrm{M}_{\sigma_{L}-}\left(\omega, 2^{-n}\right)+2^{n+L}\right)$. Consider the event $E$ that the inequality $\mathrm{M}_{\sigma_{L}-}\left(\omega, 2^{-n}\right)>n^{1.5} 2^{2 n}$ holds for infinitely many $n$. (This is the complementary event to the event that we are proving to be almost certain.) For each $n \in \mathbb{N}$ let $V_{t}^{n}$ be a nonnegative simple capital process such that $V_{0}^{n} \leq 2^{L}+c$ and $V_{\sigma_{L}}^{n} \geq 2^{-2 n-L-1} \mathrm{M}_{\sigma_{L}-}\left(\omega, 2^{-n}\right)$ (see Lemma 7 ). Then, for infinitely many $n, V_{\sigma_{L}}^{n} \geq 2^{-L-1} n^{1.5}$ on $E$. To see that the event $E$ is null, it suffices to notice that the process $\sum_{n} n^{-1.5} V^{n}$ starts from $V_{0}<\infty$ and satisfies $V_{\sigma_{L}}=\infty$ on $E$. 


\section{Proof of Theorem 1 (for $\psi=c$ )}

Let $L \in \mathbb{N}$. For all $\omega \in \Omega_{c}$ and for $t:=\sigma_{L}(\omega)$, the infinite sum in 4.8 can be bounded above as follows (cf. 4.7)):

$$
\begin{aligned}
& \sum_{k=0}^{\infty}\left(S_{\tau_{k+1}^{n} \wedge \sigma_{L}}^{n}-S_{\tau_{k}^{n} \wedge \sigma_{L}}^{n}\right)^{2} \\
& \quad=2^{2} \sum_{k=0}^{\infty}\left(\omega\left(\tau_{k}^{n} \wedge \sigma_{L}\right)-\omega\left(\tau \wedge \sigma_{L}\right)\right)^{2}\left(\omega\left(\tau_{k+1}^{n} \wedge \sigma_{L}\right)-\omega\left(\tau_{k}^{n} \wedge \sigma_{L}\right)\right)^{2} \\
& \quad \leq 2^{6-2 n} \sum_{k=0}^{\infty}\left(\omega\left(\tau_{k+1}^{n} \wedge \sigma_{L}\right)-\omega\left(\tau_{k}^{n} \wedge \sigma_{L}\right)\right)^{2}
\end{aligned}
$$

Fix an $\omega \in \Omega_{c}$ such that, from some $n \in \mathbb{N}$ on, $\mathrm{M}_{\sigma_{L}-}\left(\omega, 2^{-n}\right) \leq n^{2} 2^{2 n}$ and $\mathrm{D}_{\sigma_{L}-}\left(\omega, 2^{-n}\right) \leq n^{2} 2^{2 n}$; by Corollary 1 , this condition is satisfied for typical $\omega \in \Omega_{c}$. Let $N \in\{2,3, \ldots\}$ be so large that $\mathrm{M}_{\sigma_{L}-}\left(\omega, 2^{-n}\right) \leq n^{2} 2^{2 n}$ and $\mathrm{D}_{\sigma_{L}-}\left(\omega, 2^{-n}\right) \leq n^{2} 2^{2 n}$ for all $n \geq N$. In particular, for all $m \geq N$ :

- the number of $k \in \mathbb{N}_{0}$ such that $\tau_{k}^{m}<\infty$ does not exceed $2 m^{2} 2^{2 m}+2 \leq$ $3 m^{2} 2^{2 m}$

- the number of jumps of $\omega$ of size $2^{-m+1}$ or more over the time interval $\left[0, \sigma_{L}(\omega)\right)$ does not exceed $2 m^{2} 2^{2 m}$. (By the size of a jump of $\omega$ at $t \in$ $(0, T]$ we mean its absolute value $|\Delta \omega(t)|$.

We will write $t \in \tau^{n}$ to mean that $t$ is an element of the partition $\tau^{n}$ : there exists $k \in \mathbb{N}_{0}$ such that $t=\tau_{k}^{n}$.

Suppose $n \geq N$. We will bound different addends in (4.11) in different ways:

(A) If $\tau_{k}^{n} \leq \sigma_{L} \leq \tau_{k+1}^{n}$, we use the trivial bound

$$
\left|\omega\left(\tau_{k+1}^{n} \wedge \sigma_{L}\right)-\omega\left(\tau_{k}^{n} \wedge \sigma_{L}\right)\right| \leq 2^{1-n}+c .
$$

There are at most two such addends. We ignore the zero addends for which $\tau_{k}^{n}>\sigma_{L}$, and so assume $\tau_{k+1}^{n}<\sigma_{L}$ in the rest of this list.

(B) If $\tau_{k+1}^{n} \notin \tau^{n-1}$,

$$
\left|\omega\left(\tau_{k+1}^{n} \wedge \sigma_{L}\right)-\omega\left(\tau_{k}^{n} \wedge \sigma_{L}\right)\right| \leq 2^{2-n} .
$$

The number of such $k$ is at most $3 n^{2} 2^{2 n}$.

(C) If $\tau_{k+1}^{n} \in \tau^{n-1}$ and the size of the jump of $\omega$ at $\tau_{k+1}^{n}$ is below $2^{-n+1}$,

$$
\left|\omega\left(\tau_{k+1}^{n} \wedge \sigma_{L}\right)-\omega\left(\tau_{k}^{n} \wedge \sigma_{L}\right)\right| \leq 2^{1-n}+2^{-n+1}=2^{2-n} .
$$

The number of such $k$ is at most $3 n^{2} 2^{2 n}$. 
(D) If $\tau_{k+1}^{n} \in \tau^{n-1}$ and the size of the jump of $\omega$ at $\tau_{k+1}^{n}$ belongs to $\left[2^{-m+1}, 2^{-m+2}\right)$, where $m \in\{n, n-1, \ldots, N\}$,

$$
\left|\omega\left(\tau_{k+1}^{n} \wedge \sigma_{L}\right)-\omega\left(\tau_{k}^{n} \wedge \sigma_{L}\right)\right| \leq 2^{1-n}+2^{-m+2} .
$$

The number of such $k$ is at most $2 m^{2} 2^{2 m}$.

(E) If $\tau_{k+1}^{n} \in \tau^{n-1}$ and the size of the jump of $\omega$ at $\tau_{k+1}^{n}$ is $2^{-N+2}$ or more, we will use the trivial bound

$$
\left|\omega\left(\tau_{k+1}^{n} \wedge \sigma_{L}\right)-\omega\left(\tau_{k}^{n} \wedge \sigma_{L}\right)\right| \leq 2^{1-n}+c .
$$

The number of such $k$ is bounded by a constant $C$ (it is a constant in the sense of not depending on $n$, but it depends on $\omega$ and $L$ ).

Now we can bound the last sum in (4.11) as follows:

$$
\begin{aligned}
& \sum_{k=0}^{\infty}\left(\omega\left(\tau_{k+1}^{n} \wedge \sigma_{L}\right)-\omega\left(\tau_{k}^{n} \wedge \sigma_{L}\right)\right)^{2} \leq 2\left(2^{1-n}+c\right)^{2}+3 n^{2} 2^{2 n}\left(2^{2-n}\right)^{2} \\
+ & 3 n^{2} 2^{2 n}\left(2^{2-n}\right)^{2}+\sum_{m=N}^{n} 2 m^{2} 2^{2 m}\left(2^{1-n}+2^{-m+2}\right)^{2}+C\left(2^{1-n}+c\right)^{2} \leq 2^{-6} n^{4},
\end{aligned}
$$

the last inequality being true from some $n$ on. Remembering the definitions (4.8) and 4.9. of $U_{t}^{n}$ and $\sigma^{n}$, we can see that, from some $n$ on, $\sigma^{n}(\omega)>\sigma_{L}(\omega)$ and, therefore,

$$
U_{\sigma^{n} \wedge t}^{n}(\omega)=U_{t}^{n}(\omega) \geq\left(S_{t}^{n}(\omega)\right)^{2}
$$

for all $t \leq \sigma_{L}(\omega)$.

We have shown that, for typical $\omega \in \Omega_{c}$, from some $n$ on, 4.12 holds for all $t \leq \sigma_{L}(\omega)$. On the other hand, for typical $\omega \in \Omega_{c}$ from some $n$ on we will have

$$
\forall t \in\left[0, \sigma_{L}(\omega)\right]: U_{\sigma^{n} \wedge t}^{n}(\omega)<n^{6} 2^{-2 n} .
$$

Indeed, if $V_{t}^{n}$ is the process $U_{\sigma_{L} \wedge \sigma^{n} \wedge t}^{n}$ stopped when it reaches the value $n^{6} 2^{-2 n}$, the nonnegative capital process

$$
V_{t}:=\sum_{n=1}^{\infty} n^{-6} 2^{2 n} V_{t}^{n}
$$

will have a finite initial value and satisfy $V_{T}(\omega)=\infty$ for $\omega$ such that, for infinitely many $n, \exists t \in\left[0, \sigma_{L}(\omega)\right]: U_{\sigma^{n} \wedge t}^{n}(\omega) \geq n^{6} 2^{-2 n}$. Combining 4.12) and 4.13, we obtain that, for typical $\omega \in \Omega_{c}$, from some $n$ on, we have $\left|S_{t}^{n}\right|<n^{3} 2^{-n}$ for all $t \in\left[0, \sigma_{L}(\omega)\right]$.

We can see that, for typical $\omega \in \Omega_{c}$, the uniform distance between $A^{n-1}$ and $A^{n}$ does not exceed $n^{3} 2^{-n}$ from some $n$ on provided $\sigma_{L}=T$. Therefore, for typical $\omega \in \Omega_{c}$ the sequence $A^{n}$ is convergent in the uniform metric provided $\sigma_{L}=T$. Since the union of countably many null sets is a null set, we can omit "provided $\sigma_{L}=T "$. 


\section{Multidimensional case}

The goal of this section is to establish the existence of quadratic covariation between different price paths. We will be using a standard expression of quadratic covariation in terms of quadratic variation (the "polarization identity", used in [6], Remark 1 on p. 147).

Let $\omega^{m} \in \Omega_{\psi}, m=1, \ldots, M$. In our informal discussions we will assume that $\omega^{m}, m=1, \ldots, M$, are the price paths of all securities traded in a financial market. We will write $\omega$ for the function whose value $\omega(t)$ at time $t \in[0, T]$ is the vector $\left(\omega^{1}(t), \ldots, \omega^{M}(t)\right) \in \mathbb{R}^{M}$; the set of all such functions $\omega$ with components $\omega^{m} \in \Omega_{\psi}$ will be denoted $\Omega_{\psi}^{M}$.

In this section we set $\Omega:=\Omega_{\psi}^{M}$ (little, however, will depend on this specific definition of $\Omega$, and the definitions and statements below work for a wide class of $\Omega$ ). The $\sigma$-algebra $\mathcal{F}_{t}^{\circ}, t \in[0, T]$, is the smallest $\sigma$-algebra on $\Omega$ that makes all functions $\omega \in \Omega \mapsto \omega^{m}(s)$, where $m \in\{1, \ldots, M\}$ and $s \in[0, t]$, measurable. The $\sigma$-algebras $\mathcal{F}_{t}$, processes, stopping times $\tau$, and $\sigma$-algebras $\mathcal{F}_{\tau}$ on $\Omega$ are defined for the sample space $\Omega$ in the same way as for the sample space $\Omega_{\psi}$ : replacing $\Omega_{\psi}$ by $\Omega$ is the only change. The other definitions of Section 3 carry over to the case of $\Omega=\Omega_{\psi}^{M}$, with the following changes. In the definition of simple trading strategies, the bounded $\mathcal{F}_{\tau_{n}}$-measurable functions $h_{n}$ now take values in $\mathbb{R}^{M}$, and the definition 3.2 of a simple capital process now becomes

$$
\mathcal{K}_{t}^{G, \alpha}(\omega):=\alpha+\sum_{n=1}^{\infty} h_{n}(\omega) \cdot\left(\omega\left(\tau_{n+1} \wedge t\right)-\omega\left(\tau_{n} \wedge t\right)\right), \quad t \in[0, T]
$$

where "." stands for dot product in $\mathbb{R}^{M}$.

For all $\omega \in \Omega$ and $n \in \mathbb{N}_{0}$, let us define a sequence $\tau_{k}^{n}(\omega), k=0,1,2, \ldots$, as the finite set

$$
\begin{aligned}
\left\{\tau_{k}^{n}\left(\omega^{m}\right) \mid k \in \mathbb{N}_{0}, m \in\{1, \ldots, M\}\right\} & \\
& \cup\left\{\tau_{k}^{n}\left(\omega^{m}+\omega^{l}\right) \mid k \in \mathbb{N}_{0}, m \in\{1, \ldots, M\}, l \in\{1, \ldots, M\}\right\}
\end{aligned}
$$

ordered in the increasing order (with repetitions removed, by the definition of a set); to make the sequence $\tau^{n}(\omega)$ infinite, we complement it by $\infty, \infty, \ldots$ on the right. It is clear that $\tau_{k}^{n}(\omega)$ as functions of $\omega \in \Omega$ are stopping times.

The sequence $\left(\tau_{0}^{n}(\omega), \tau_{1}^{n}(\omega), \ldots\right)$ will be denoted $\tau^{n}(\omega)$; for a fixed $\omega$, this is a partition of $[0, T]$. By $\tau(\omega)$ we denote the sequence of partitions $\tau^{n}(\omega)$, $n \in \mathbb{N}_{0}$.

Theorem 2. For typical $\omega \in \Omega=\Omega_{\psi}^{M}$, each $\omega^{m}, m \in\{1, \ldots, M\}$, has quadratic variation along $\tau(\omega)$, and each $\omega^{m}+\omega^{l},(m, l) \in\{1, \ldots, M\}^{2}$, has quadratic variation along $\tau(\omega)$.

Before proving Theorem 2, let us see how to use it to define quadratic covariation. We will use the notation $\left[\omega^{m}\right]$ for the quadratic variation of $\omega^{m}, m \in\{1, \ldots, M\}$, and $\left[\omega^{m}+\omega^{l}\right]$ for the quadratic variation of $\omega^{m}+\omega^{l}$, 
$(m, l) \in\{1, \ldots, M\}^{2}$, along $\tau(\omega)$. Now we define the quadratic covariation processes between different price paths by

$$
[\omega]_{t}^{m, l}:=\frac{1}{2}\left(\left[\omega^{m}+\omega^{l}\right]_{t}-\left[\omega^{m}\right]_{t}-\left[\omega^{l}\right]_{t}\right), \quad t \in[0, T],(m, l) \in\{1, \ldots, M\}^{2} ;
$$

they exist for typical $\omega \in \Omega$. Notice that $[\omega]^{m, m}=\left[\omega^{m}\right]$ and that

$$
[\omega]_{t}^{m, l}=\left([\omega]^{m, l}\right)_{t}^{\mathrm{c}}+\sum_{s \in(0, t]} \Delta \omega^{m}(s) \Delta \omega^{l}(s)
$$

where $f^{c}$ stands for the continuous part of a function $f$.

\section{Proof of Theorem 2}

We have already proved that $\omega^{m}$ have quadratic variation, but along a different sequence of partitions, $\tau\left(\omega^{m}\right)$ rather than $\tau(\omega)$. Now we stop and change our positions as soon as any security in the market or the sum of two securities significantly change their value, so we have one sequence of partitions for the whole market. But the argument of Section 4 still works, as it depends on relatively few properties of the sequence of partitions, which are still satisfied.

To see what properties of the array $\tau_{k}^{n}$ of stopping times were used in Section 4 let $\iota_{k}^{n}\left(n \in \mathbb{N}_{0}, k \in \mathbb{N}_{0}\right)$ be a general array of stopping times on $\Omega$ of the same kind as $\tau_{k}^{n}: \iota^{n}(\omega), n \in \mathbb{N}_{0}$, are nested partitions of $[0, T]$ for each $\omega \in \Omega$ and all $\iota_{k}^{n}$ are stopping times; in particular, $0=\iota_{0}^{n} \leq \iota_{1}^{n} \leq \cdots$ and all $\iota_{k}^{n}$ take values in $[0, T] \cup\{\infty\}$. We say that the sequence $\iota$ of random (i.e., depending on $\omega \in \Omega$ ) nested partitions $\iota^{n}$ is of dyadic type for a process $W$ (such as $\omega^{m}$ or $\omega^{m}+\omega^{l}$ ) if there exist a polynomial $p$ and a constant $C>0$ such that:

(a) For all $\omega \in \Omega$, all $n \in \mathbb{N}_{0}$, and all $0 \leq s<t \leq T$ such that $\left|W_{t}-W_{s}\right|>$ $C 2^{-n}$, there exists $k$ such that $\iota_{k}^{n}(\omega) \in(s, t]$.

(b) For typical $\omega \in \Omega$, from some $n$ on, the number of $k$ such that $\iota_{k}^{n}(\omega)<\infty$ is at most $p(n) 2^{2 n}$.

Intuitively, (a) says that the partitions are sufficiently fine, and (b) says that they are not too fine. Condition (a) implies that $\iota(\omega)$ should exhaust $\omega$ and can be regarded as a quantitative version of this requirement.

A process $W$ has moderate jumps if there exists a nondecreasing function $\phi:[0, \infty) \rightarrow(0, \infty)$ such that, for each $\omega \in \Omega$,

$$
\forall t \in(0, T]:\left|\Delta W_{t}(\omega)\right| \leq \phi\left(\sup _{s \in[0, t)}\left|W_{s}(\omega)\right|\right)
$$

(cf. (3.1)). We let $W(\omega)$, where $W$ is a process and $\omega \in \Omega$, stand for the function $t \in[0, T] \mapsto W_{t}(\omega) \in \mathbb{R}$. The quadratic variation of $W(\omega)$ along $\iota(\omega)$ is defined 
as before (cf. 2.1) and (4.2) with $W(\omega)$ in place of $\omega$, namely, as the uniform limit as $n \rightarrow \infty$ of

$$
A_{t}^{n, \tau}(\omega):=\sum_{k=1}^{\infty}\left(W_{\iota_{k}^{n} \wedge t}(\omega)-W_{\iota_{k-1}^{n} \wedge t}(\omega)\right)^{2} .
$$

We saw in Section 4 that the sequence $\tau(\omega)$ of partitions defined earlier in this section is of dyadic type for all $\omega \in \Omega$ (with $C:=2$ and $p(n):=\left(M^{2}+M\right) 3 n^{2}$ sufficient). This shows, in combination with Proposition 2 below, that the following proposition generalizes Theorem 1 .

Proposition 1. If an array $\iota_{k}^{n}$ of stopping times on $\Omega$ is of dyadic type for a simple capital process $W$ that has moderate jumps, $W(\omega)$ has quadratic variation along $\iota(\omega)$ for typical $\omega \in \Omega$.

Proof. It is easy to check that the proof of Theorem 1. with suitable changes, still works in our current more general situation; now we consider paths $W(\omega)$ instead of $\omega$.

Condition (a) (with $C=2$ ) was used in establishing the nonnegativity of the simple capital process (4.8) over $\left[0, \sigma^{n} \wedge T\right]$ and our upper bound on the infinite sum (4.11) in 4.8). For our arguments to go through, the definition of the simple capital process 4.8 should be modified: the addend $2^{-2 n+8} c^{2}$ should be replaced by a constant (depending only on $c$ and $C$ ) times $2^{-2 n}$, and the polynomial $n^{4}$ in the addend $n^{4} 2^{-2 n}$ should be replaced by a different polynomial (depending on $p$ ). To upper bound 4.11), we have used (in the cases (A) (E) of Section 4 the inequality

$$
\left|\omega\left(\tau_{k+1}^{n}(\omega)\right)-\omega\left(\tau_{k}^{n}(\omega)\right)\right| \leq 2^{1-n}+\left|\Delta \omega\left(\tau_{k+1}^{n}\right)\right|
$$

assuming $\tau_{k+1}^{n}(\omega)<\sigma_{L}(\omega)$. This inequality continues to hold with $2^{1-n}$ replaced by $C 2^{-n}$ in our current situation: indeed, condition (a) implies that

$$
\left|\omega\left(\iota_{k+1}^{n}(\omega)-\right)-\omega\left(\iota_{k}^{n}(\omega)\right)\right| \leq C 2^{-n} .
$$

Condition (b) can be used in the cases (B) (D) in Section 4 to bound the number of $k$ covered by each of those cases. We need the weaker requirement that $\iota_{k}^{n}<\infty$ for only finitely many $k$ also to satisfy a requirement in the definition of simple trading strategies.

Let $m, l \in\{1, \ldots, M\}$. The processes $\omega^{m}$ and $\omega^{m}+\omega^{l}$ have moderate jumps (with $\phi:=\psi$ and $\phi:=2 \psi$, respectively). Both $\omega^{m}$ and $\omega^{m}+\omega^{l}$ are simple capital processes. We can see that, for typical $\omega \in \Omega, \omega^{m}$ and $\omega^{m}+\omega^{l}$ have quadratic variation along $\tau(\omega)$.

The proof of Theorem 2 is now complete. However, there remains the question of invariance of our definitions; e.g., is the quadratic variation $A^{\tau(\omega)}\left(\omega^{m}\right)$ of $\omega^{m}$ along $\tau(\omega)$ (our new definition) the same function as the quadratic variation $A^{\tau\left(\omega^{m}\right)}\left(\omega^{m}\right)$ of $\omega^{m}$ along $\tau\left(\omega^{m}\right)$ (our old definition)? The proof of Theorem 1 shows that they are. For simplicity, we will only spell out the argument in the framework of Proposition 1 . 
Proposition 2. If arrays $\iota_{k}^{n}$ and $\kappa_{k}^{n}$ of stopping times on $\Omega$ are of dyadic type for a simple capital process $W, W(\omega)$ has the same quadratic variation along $\iota(\omega)$ and along $\kappa(\omega)$ for typical $\omega \in \Omega$.

Proof. Without loss of generality we can assume that $\iota$ is nested in $\kappa$ : for all $\omega \in \Omega, n \in \mathbb{N}_{0}$, and $k \in \mathbb{N}_{0}$, there exists $k^{\prime} \in \mathbb{N}_{0}$ such that $\kappa_{k^{\prime}}^{n}(\omega)=\iota_{k}^{n}(\omega)$. (Indeed, this special case will imply that general $\iota$ and $\kappa$ lead to the same quadratic variation as their union.) The argument in the proof of Theorem 1 (applied to $\iota(\omega)$ and $\kappa(\omega)$ in place of $\tau^{n-1}(\omega)$ and $\tau^{n}(\omega)$, so that $S_{t}^{n}(\omega):=$ $\left.A_{t}^{n, \kappa}(\omega)-A_{t}^{n, \iota}(\omega)\right)$ shows that, for typical $\omega \in \Omega$, the uniform distance between $A^{n, \iota}(\omega)$ and $A^{n, \kappa}(\omega)$ converges to 0 (exponentially fast) as $n \rightarrow \infty$. Therefore, the uniform limits of $A^{n, L}(\omega)$ and $A^{n, \kappa}(\omega)$ coincide.

\section{Föllmer's and Norvaiša's quadratic variation}

In this section we adapt Föllmer's [6] and Norvaiša's [14 definitions of quadratic variation to our framework (in particular, to our bounded time interval $[0, T]$ ). Let $\omega \in D[0, T]$ and $\pi$ be a nested sequence of partitions that exhausts $\omega$.

We say that $\omega$ has Föllmer's quadratic variation along $\pi$ if the sequence of finite measures

$$
\xi^{n}:=\sum_{k=1}^{\infty}\left(\omega\left(\pi_{k}^{n} \wedge T\right)-\omega\left(\pi_{k-1}^{n} \wedge T\right)\right)^{2} \delta_{\omega\left(\pi_{k-1}^{n} \wedge T\right)}
$$

( $\delta_{t}$ being the Dirac measure at $t$ ) on $[0, T]$ weakly converges to a finite measure $\xi$ on $[0, T]$ whose discrete part is given by the squared jumps of $\omega$ :

$$
V(t)=V^{\mathrm{c}}(t)+\sum_{s \in(0, t]}(\Delta \omega(s))^{2},
$$

where $V$ is the distribution function of $\xi$ and $V^{\mathrm{c}}$ is its continuous part. We will say that $V$ is Föllmer's quadratic variation of $\omega$ along $\pi$.

Föllmer's [6] original definition of quadratic variation is different from the definition above in the following respects: it does not require the sequence $\pi$ of partitions to be nested; it does not require $\pi$ to exhaust $\omega$; it requires $\pi$ to be dense; it assumes the unbounded time interval $[0, \infty)$. Föllmer uses the notation $[\omega, \omega]$ for the function $V$ and does not use the term "quadratic variation" in respect of $V$.

We say that $\omega$ has Norvais̆a's quadratic variation along $\pi$ if there exists a function $V \in D[0, T]$ such that, for all $0 \leq s<t \leq T$,

$$
V(t)-V(s)=\lim _{n \rightarrow \infty} \sum_{k=1}^{\infty}\left(\omega\left(s \vee \pi_{k}^{n} \wedge t\right)-\omega\left(s \vee \pi_{k-1}^{n} \wedge t\right)\right)^{2}
$$

and, for any $t \in(0, T]$,

$$
\Delta V(t)=(\Delta \omega(t))^{2} .
$$


The function $V$ is called Norvaiša's quadratic variation of $\omega$ along $\pi$.

Norvaiša's (14, p. 1) original definition is different from the definition in the previous paragraph in the following respects: it does not require $\pi$ to exhaust $\omega$; it requires $\pi$ to be dense; it only requires $\omega$ to be a regulated, rather than càdlàg, function. Norvaiša calls $V$ the bracket function of $\omega$.

We can also weaken Norvaiša's requirements to the function $V$. Namely, we will say that $\omega$ has weak quadratic variation along $\pi$ if there exists a function $V \in D[0, T]$ such that

$$
V(t)=\lim _{n \rightarrow \infty} \sum_{k=1}^{\infty}\left(\omega\left(\pi_{k}^{n} \wedge t\right)-\omega\left(\pi_{k-1}^{n} \wedge t\right)\right)^{2}
$$

at all points $t \in[0, T]$ of continuity of $V$ and $\sqrt[6.3)]{ }$ holds for all $t \in(0, T]$. The function $V$ is then called the weak quadratic variation of $\omega$ along $\pi$.

The last two notions of quadratic variation are equivalent to the one defined in Section 2:

Proposition 3. Let $\omega \in D[0, T]$ and $\pi$ be a nested sequence of partitions that exhausts $\omega$. The following three conditions are equivalent:

(a) the quadratic variation of $\omega$ along $\pi$ exists (in the sense of Section 2) and is $V=A^{\pi}$;

(b) Norvaiša's quadratic variation of $\omega$ along $\pi$ exists and is $V$;

(c) the weak quadratic variation of $\omega$ along $\pi$ exists and is $V$.

Proof. First we assume condition (a) and prove condition (b) We know that 6.2 (with $V:=A^{\pi}$ ) holds for $s=0$, so we assume $s>0$. In this case, 6.2 is equivalent to

$$
\begin{aligned}
\left(\omega\left(\pi_{\bar{k}(s, n)}^{n}\right)-\omega\left(\pi_{\underline{k}(s, n)}^{n}\right)\right)^{2}- & \left(\omega(s)-\omega\left(\pi_{\underline{k}(s, n)}^{n}\right)\right)^{2} \\
& -\left(\omega\left(\pi_{\bar{k}(s, n)}^{n}\right)-\omega(s)\right)^{2} \rightarrow 0 \quad(n \rightarrow \infty),
\end{aligned}
$$

where $\underline{k}(s, n)$ is the largest $k$ satisfying $\pi_{k}^{n} \leq s$ and $\bar{k}(s, n)$ is the smallest $k$ satisfying $\pi_{k}^{n} \geq s(6.5)$ assumes that $\omega$ is not constant over $[s, t]$; the simple case where it is has to be considered separately). We can rewrite (6.5) as

$$
\left(\omega\left(\pi_{\bar{k}(s, n)}^{n}\right)-\omega(s)\right)\left(\omega(s)-\omega\left(\pi_{\underline{k}(s, n)}^{n}\right)\right) \rightarrow 0,
$$

which immediately follows from Lemma 1 if $s \in(\underline{k}(s, n), \bar{k}(s, n))$ for all $n$ (this is the non-trivial case), the first factor stays bounded and the second tends to 0 as $n \rightarrow \infty$. This proves 6.2 , and $(6.3)$ holds by Lemma 2 .

It is obvious that (b) implies (c).

Finally, we assume condition (c) and prove (a). We know that $A^{n, \pi} \rightarrow V$ at all points of continuity of $V$ as $n \rightarrow \infty$, and our goal is to prove that $A^{n, \pi} \rightarrow V$ 
uniformly. By the compactness of $[0, T]$, it suffices to prove that, for each point $t^{*} \in[0, T], A^{n, \pi} \rightarrow V$ uniformly in some neighbourhood of $t^{*}$. Fix such $t^{*}$. We consider two cases separately:

- Suppose $\Delta V\left(t^{*}\right)=0$. Let $\epsilon \in(0,1)$. Choose $t^{\prime}<t^{*}$ and $t^{\prime \prime}>t^{*}$ such that $\Delta V\left(t^{\prime}\right)=0, \Delta V\left(t^{\prime \prime}\right)=0$, and $V(t)$ belongs to $\left(V\left(t^{*}\right)-\epsilon, V\left(t^{*}\right)+\epsilon\right)$ for all $t \in\left[t^{\prime}, t^{\prime \prime}\right]$. From some $n$ on, $A_{t^{\prime}}^{n, \pi}$ belongs to $\left(V\left(t^{\prime}\right)-\epsilon, V\left(t^{\prime}\right)+\epsilon\right)$ and $A_{t^{\prime \prime}}^{n, \pi}$ belongs to $\left(V\left(t^{\prime \prime}\right)-\epsilon, V\left(t^{\prime \prime}\right)+\epsilon\right)$. Therefore, from some $n$ on, both $A_{t^{\prime}}^{n, \pi}$ and $A_{t^{\prime \prime}}^{n, \pi}$ belong to $\left(V\left(t^{*}\right)-2 \epsilon, V\left(t^{*}\right)+2 \epsilon\right)$. From the proof of Lemma 2 we know that, from some $n$ on, $A_{t}^{n, \pi}$ never drops by more than $\epsilon$ as $t$ increases (see 2.3), which we show to be impossible from some $n$ on when $t_{1}<t_{2}$; the simple argument in that proof only depends on conditions that are satisfied in our current context). Therefore, from some $n$ on, $A_{t}^{n, \pi}$ belongs to $\left(V\left(t^{*}\right)-3 \epsilon, V\left(t^{*}\right)+3 \epsilon\right)$ for all $t \in\left[t^{\prime}, t^{\prime \prime}\right]$. We can see that, from some $n$ on, $A_{t}^{n, \pi}$ belongs to $(V(t)-4 \epsilon, V(t)+4 \epsilon)$ for all $t \in\left[t^{\prime}, t^{\prime \prime}\right]$.

- Suppose that $\Delta V\left(t^{*}\right) \neq 0$. Let $\epsilon \in(0,1)$. Choose $t^{\prime}<t^{*}$ and $t^{\prime \prime}>t^{*}$ such that $\Delta V\left(t^{\prime}\right)=0, \Delta V\left(t^{\prime \prime}\right)=0, V(t)$ belongs to the interval $\left(V\left(t^{*}-\right)-\right.$ $\left.\epsilon, V\left(t^{*}-\right)+\epsilon\right)$ for all $t \in\left[t^{\prime}, t^{*}\right)$, and $V(t)$ belongs to $\left(V\left(t^{*}\right)-\epsilon, V\left(t^{*}\right)+\epsilon\right)$ for all $t \in\left[t^{*}, t^{\prime \prime}\right]$. From some $n$ on,

$$
\begin{aligned}
& A_{t^{\prime}}^{n, \pi} \in\left(V\left(t^{*}-\right)-2 \epsilon, V\left(t^{*}-\right)+2 \epsilon\right), \\
& A_{t^{\prime \prime}}^{n, \pi} \in\left(V\left(t^{*}\right)-2 \epsilon, V\left(t^{*}\right)+2 \epsilon\right) .
\end{aligned}
$$

From some $n$ on there is a $k$ such that $t^{*}=\pi_{k}^{n}$ and $\pi_{k-1}^{n} \in\left(t^{\prime}, t^{*}\right)$ (unless $\omega$ is constant over $\left(t^{\prime}, t^{*}\right)$, in which case $\pi_{k-1}^{n}$ should be replaced by $t^{\prime}$ in the rest of this proof); and from some $n$ on, $\operatorname{osc}_{\pi^{n}}(\omega)<\epsilon$ (Lemma 1). Since $\Delta V\left(t^{*}\right)=\left(\Delta \omega\left(t^{*}\right)\right)^{2}$, for such $n$ we have

$$
A_{\pi_{k}^{n}}^{n, \pi}-A_{\pi_{k-1}^{n}}^{n, \pi}=\left(\omega\left(t^{*}\right)-\omega\left(\pi_{k-1}^{n}\right)\right)^{2}>\Delta V\left(t^{*}\right)-2 \Delta_{\omega} \epsilon,
$$

where $\Delta_{\omega}:=\sup _{t \in(0, T]}|\Delta \omega(t)|$. We know that, from some $n$ on, $A_{t}^{n, \pi}$ never drops by more than $\epsilon$ as $t$ increases. According to (6.6), 6.7), and 6.8, we then have

$$
\left\{\begin{array}{l}
A_{\pi_{k}^{n}}^{n, \pi}-A_{\pi_{k-1}^{n}}^{n, \pi}>\Delta V\left(t^{*}\right)-2 \Delta_{\omega} \epsilon \\
A_{\pi_{k}^{n}}^{n, \pi}<V\left(t^{*}\right)+3 \epsilon \\
A_{\pi_{k-1}^{n}}^{n, \pi}>V\left(t^{*}-\right)-3 \epsilon .
\end{array}\right.
$$

This system of three inequalities immediately implies

$$
\left\{\begin{array}{l}
A_{\pi_{k}^{n}}^{n, \pi}>V\left(t^{*}\right)-2 \Delta_{\omega} \epsilon-3 \epsilon \\
A_{\pi_{k-1}^{n}}^{n, \pi}<V\left(t^{*}-\right)+2 \Delta_{\omega} \epsilon+3 \epsilon .
\end{array}\right.
$$

Combining this with (6.6), 6.7), and $\operatorname{osc}_{\pi^{n}}(\omega)<\epsilon$, we can see that

$$
\begin{cases}A_{t}^{n, \pi} \in\left(V\left(t^{*}\right)-2 \Delta_{\omega} \epsilon-4 \epsilon, V\left(t^{*}\right)+3 \epsilon\right) & \text { if } t \in\left[t^{*}, t^{\prime \prime}\right] \\ A_{t}^{n, \pi} \in\left(V\left(t^{*}-\right)-3 \epsilon, V\left(t^{*}-\right)+2 \Delta_{\omega} \epsilon+4 \epsilon\right) & \text { if } t \in\left[t^{\prime}, t^{*}\right) .\end{cases}
$$


Therefore, from some $n$ on, we have

$$
\left|A_{t}^{n, \pi}-V(t)\right|<2 \Delta_{\omega} \epsilon+5 \epsilon
$$

for all $t \in\left[t^{\prime}, t^{\prime \prime}\right]$.

In both cases $A^{n, \pi}$ converges to $V$ uniformly in some neighbourhood of $t^{*}$, which completes the proof.

On the other hand, Föllmer's notion of quadratic variation is different and even anomalous unless $\pi$ is dense. Set, e.g.,

$$
\omega(t):= \begin{cases}0 & \text { if } t \in[0, T / 2) \\ 1 & \text { if } t \in[T / 2, T) \\ 0 & \text { if } t=T\end{cases}
$$

and consider the sequence of nested partitions

$$
\pi^{1}=\pi^{2}=\cdots:=(0, T / 2, T, \infty, \infty, \ldots),
$$

which exhausts $\omega$. The three definitions whose equivalence is asserted in Proposition 3 give the same quadratic variation $V$ of $\omega$ along $\pi$,

$$
V(t):= \begin{cases}0 & \text { if } t \in[0, T / 2) \\ 1 & \text { if } t \in[T / 2, T) \\ 2 & \text { if } t=T\end{cases}
$$

whereas Föllmer's does not exist: the first part of the definition gives

$$
V(t):= \begin{cases}1 & \text { if } t \in[0, T / 2) \\ 2 & \text { if } t \in[T / 2, T],\end{cases}
$$

which fails to satisfy 6.1 at $t=T(V(0) \neq 0$ also looks anomalous). The anomalies disappear when $\pi$ is dense:

Proposition 4. Let $\omega \in D[0, T]$ and $\pi$ be a dense nested sequence of partitions.

(a) If the quadratic variation $V$ of $\omega$ along $\pi$ exists in the sense of any of the three definitions of Proposition 3, $V$ is also Fölmer's quadratic variation of $\omega$ along $\pi$.

(b) If Föllmer's quadratic variation $V$ of $\omega$ along $\pi$ exists, $V$ is also the quadratic variation of $\omega$ along $\pi$ in the sense of the three definitions of Proposition 4

Proof. We start from (a). Let $V$ be the quadratic variation of $\omega$ along $\pi$ in the sense of the definitions of Proposition 3 Since 6.1 is obviously equivalent 
to (6.3), the definition of Föllmer's quadratic variation shows that it suffices to prove

$$
\sum_{k \in \mathbb{N}: \pi_{k-1}^{n} \leq t}\left(\omega\left(\pi_{k}^{n} \wedge T\right)-\omega\left(\pi_{k-1}^{n}\right)\right)^{2} \rightarrow V(t)
$$

as $n \rightarrow \infty$, where $t \in[0, T]$ is such that $V$ is continuous at $t$, i.e., by 6.3 , $\omega$ is continuous at $t$. Comparing (6.9) with 6.4 , we can see that, furthermore, it suffices to prove

$$
\left(\omega(t)-\omega\left(\pi_{\underline{k}(t, n)}^{n}\right)\right)^{2}-\left(\omega\left(\pi_{\underline{k}(t, n)+1}^{n} \wedge T\right)-\omega\left(\pi_{\underline{k}(t, n)}^{n}\right)\right)^{2} \rightarrow 0,
$$

which immediately follows from the continuity of $\omega$ at $t$ provided $\pi$ is dense.

The argument in the previous paragraph (cf. 6.10) also establishes (b).

Remark 4. Föllmer's [6] and Norvaiša's [14 condition that the sequence of partitions $\pi$ should be dense would not in fact be a big obstacle in this paper: to make the random sequence of partitions $\tau^{n}$ formed by the stopping times $\tau_{k}^{n}$ (as defined in Section 4 or Section 5 dense we can simply complement $\tau^{n}(\omega)$ by the points $T k 2^{-n}, k=1, \ldots, 2^{n}-1$. The properties (a) (b) of Section 5 will be still satisfied after this extension and the sequence will be still nested. This step is, however, awkward, and we avoid it.

\section{Implications of the existence of quadratic vari- ation}

This section reviews some known implications (the simpler ones from 6 and [14]) of the existence of quadratic variation. Let $\omega \in D[0, T]$ and $\pi=\left(\pi^{n}\right)$ be a nested sequence of partitions that exhausts $\omega$. We are mainly interested in the case where $\pi=\left(\pi^{n}(\omega)\right)$ is the sequence of partitions formed by the stopping times $\tau_{k}^{n}$, as defined in Section 4 . We know that in this case the quadratic variation of $\omega$ exists unless $\omega$ is in a null set (Theorem 1). To simplify notation, we do not consider the multidimensional case (Section 5 and Theorem 2).

Throughout this section, we fix $\omega \in D[0, T]$ and $\pi=\left(\pi^{n}\right)$ such that $\pi$ is a nested sequence of partitions that exhausts $\omega$ and the quadratic variation of $\omega$ along $\pi$ exists (cf. Proposition 3). As in Section 5, we use square brackets to denote the quadratic variation along $\pi$, when it exists; e.g., $[\omega]$ is the quadratic variation of $\omega$ along $\pi$. A minor difference of the results that we state in this section from the original ones stated in [6] and [14 is that we do not assume that $\pi$ is dense: the assumption of denseness becomes redundant under our assumptions since we can always remove all intervals of constancy from the domain of $\omega$. 


\section{Stochastic integration and Itô's lemma}

Suppose $f: \mathbb{R} \rightarrow \mathbb{R}$. For each $t \in[0, T]$ and $\omega \in D[0, T]$, define

$$
\int_{0}^{t} f(\omega(s-)) \mathrm{d} \omega(s):=\lim _{n \rightarrow \infty} \sum_{k=0}^{\infty} f\left(\omega\left(\pi_{k}^{n}(\omega) \wedge t\right)\right)\left(\omega\left(\pi_{k+1}^{n} \wedge t\right)-\omega\left(\pi_{k}^{n} \wedge t\right)\right) .
$$

The following two propositions are Föllmer's (6], p. 144) theorem adapted to our framework. The first proposition says that the stochastic integral (7.1) exists.

Proposition 5. Let $f \in C^{1}(\mathbb{R})$. The limit in $\sqrt{7.1}$ exists for each $t \in[0, T]$, and as a function of $t \in[0, T]$ is an element of $D[0, T]$.

The second proposition gives the Itô-Föllmer formula.

Proposition 6. Let $F \in C^{2}(\mathbb{R})$. For all $t \in[0, T]$,

$$
\begin{aligned}
F(\omega(t))= & F(\omega(0))+\int_{0}^{t} F^{\prime}(\omega(s-)) \mathrm{d} \omega(s)+\frac{1}{2} \int_{0}^{t} F^{\prime \prime}(\omega(s)) \mathrm{d}[\omega]_{s}^{c} \\
& +\sum_{s \in(0, t]}\left(\Delta F(\omega(s))-F^{\prime}(\omega(s-)) \Delta \omega(s)\right) .
\end{aligned}
$$

For the extension of the stochastic integral and the Itô-Föllmer formula to the case of $\omega$ taking values in $\mathbb{R}^{m}$, see 6 , pp. 147-148. This extension uses the quadratic covariation processes: see (5.1).

An important development of Föllmer's results is their extension by Cont and Fournié 3 to non-anticipative functionals. The existence of the limit in (7.1) when $f\left(\omega\left(\pi_{k}^{n}(\omega) \wedge t\right)\right)$ is replaced by $g\left(\left.\omega\right|_{\left[0, \pi_{k}^{n}(\omega) \wedge t\right]}\right)$, where $g$ is a functional satisfying certain regularity conditions (including being non-anticipative), is established in Theorem 4 of [3]. The same theorem gives an Itô-Föllmer formula for non-anticipative functionals.

\section{Quadratic variation for other processes}

We now state some known results for the existence of quadratic variation for two kinds of processes different from the basic process $W_{t}(\omega):=\omega(t)$ : namely, for functions of the form $f(\omega(t))$, where $f \in C^{1}(\mathbb{R})$, and for stochastic integrals w.r. to $\omega$.

Suppose $f \in C^{1}(\mathbb{R})$. Föllmer notices in [6] (Remark 2 on p. 148) that a standard argument in the theory of stochastic integration (as in [10], Theorem VI.5 on p. 359) implies that the quadratic variation of $f(\omega)$ along $\pi$ exists and is equal to

$$
[f(\omega)]_{t}=\int_{0}^{t}\left(f^{\prime}(\omega(s))\right)^{2} \mathrm{~d}[\omega]_{s}^{\mathrm{c}}+\sum_{s \in(0, t]}(\Delta f(\omega(s)))^{2}
$$


Norvaiša's Theorem 3.26 in 14 implies that the quadratic variation of the stochastic integral $\Phi(t):=\int_{0}^{t} f(\omega(s-)) \mathrm{d} \omega(s)$ exists and is equal to

$$
[\Phi]_{t}=\int_{0}^{t} f^{2}(\omega(s-)) \mathrm{d}[\omega]_{s} .
$$

Suppose that $\omega \in D[0, T]$ is positive (cf. the second example discussed in Section 3). In this case it is natural to measure quadratic variation on the relative rather than absolute scale, and so to consider the quadratic variation of the logarithm of $\omega$. By Föllmer's result, the quadratic variation process of $\ln \omega$ is

$$
[\ln \omega]_{t}=\int_{0}^{t} \frac{\mathrm{d}[\omega]_{s}^{\mathrm{c}}}{\omega^{2}(s)}+\sum_{s \in(0, t]}(\Delta \ln \omega(s))^{2} .
$$

By Norvaiša's result, the quadratic variation process of the stochastic logarithm $\operatorname{Ln} \omega(t):=\int_{0}^{t} \frac{\mathrm{d} \omega(s)}{\omega(s-)}$ of $\omega$ is

$$
[\operatorname{Ln} \omega]_{t}=\int_{0}^{t} \frac{\mathrm{d}[\omega]_{s}}{\omega^{2}(s-)}
$$

\section{Conclusion}

In this section we discuss possible directions of further research. This paper shows the existence of quadratic variation for typical price paths in $\Omega_{\psi}$. It is easy to see that Theorem1 1 becomes false if we simply set $\psi:=\infty$, but an interesting question is whether we can set $\psi:=\infty$ if we only consider nonnegative $\omega$ (for many securities, $\omega \geq 0$ can be assumed from economic considerations).

Another possible way to get rid of the assumption that the jumps of $\omega$ are bounded by a function of $\omega$ is to allow trading in American, or binary American, options to hedge against huge jumps of $\omega$. If the prices of such out-of-the-money options tend to zero sufficiently fast as their moneyness decreases, we can expect that the analogue of Theorem 1 will continue to hold even for $\psi:=\infty$.

In Section 4 , we proved the existence of quadratic variation only for a specific array of stopping times $\left(\tau_{k}^{n}\right)$. In Section 5, we noticed that the argument of Section 4 works for the class of arrays $\left(\tau_{k}^{n}\right)$ which we called arrays of dyadic type and that any two arrays of dyadic type lead to the same values of quadratic variation for typical $\omega$. It is clear that this observation can be extended to a much wider class of arrays.

One interpretation of the stochastic integral $(7.1)$ is that it is the capital of a trading strategy. We, however, also define capital processes directly: see (3.2) and (3.3). Can all nonnegative capital processes $(3.3)$ be represented as stochastic integrals? It is clear that the "Markovian" definition (7.1) is not sufficient (the trading strategy in (7.1) takes into account only the current price), so this question is about the extension of (7.1) to non-anticipating functionals, as in [3]. 


\section{Acknowledgments}

An earlier version of this paper was the basis of my talk at the 2014 Vilnius Conference on Probability Theory and Mathematical Statistics (section "Random processes", session "Rough paths"), and I am grateful to the organizers for inviting me and to the listeners for useful comments, with special thanks to Rimas Norvaiša. This research was supported by the Air Force Office of Scientific Research (grant FA9550-14-1-0043).

\section{References}

[1] Patrick Billingsley. Convergence of Probability Measures. Wiley, New York, 1968.

[2] Michel Bruneau. Sur la p-variation des surmartingales. Séminaire de probabilités de Strasbourg, 13:227-232, 1979.

[3] Rama Cont and David-Antoine Fournié. Change of variable formulas for non-anticipative functionals on path space. Journal of Functional Analysis, 259:1043-1072, 2010.

[4] Claude Dellacherie and Paul-André Meyer. Probabilities and Potential. North-Holland, Amsterdam, 1978. Chapters I-IV. French original: 1975; reprinted in 2008.

[5] Federal Reserve Board. Credit by brokers and dealers (Regulation T). 12 CFR 220.12, 2014.

[6] Hans Föllmer. Calcul d'Itô sans probabilités. Séminaire de probabilités de Strasbourg, 15:143-150, 1981.

[7] Hans Föllmer and Alexander Schied. Stochastic Finance: An Introduction in Discrete Time. De Gruyter, Berlin, third edition, 2011.

[8] Peter Fortune. Margin requirements, margin loans, and margin rates: practice and principles. New England Economic Review, pages 19-44, September/October 2000.

[9] Andrei N. Kolmogorov. Sur la loi des grands nombres. Atti della Reale Accademia Nazionale dei Lincei. Classe di scienze fisiche, matematiche, e naturali. Rendiconti Serie VI, 185:917-919, 1929.

[10] Paul-André Meyer. Un cours sur les intégrales stochastiques. Séminaire de probabilités de Strasbourg, 10:245-400, 1976.

[11] Kenshi Miyabe and Akimichi Takemura. Convergence of random series and the rate of convergence of the strong law of large numbers in game-theoretic probability. Stochastic Processes and their Applications, 122:1-30, 2012. 
[12] Kenshi Miyabe and Akimichi Takemura. The law of the iterated logarithm in game-theoretic probability with quadratic and stronger hedges. Stochastic Processes and their Applications, 123:3132-3152, 2013.

[13] Kenshi Miyabe and Akimichi Takemura. Derandomization in gametheoretic probability. Stochastic Processes and their Applications, 2014. To appear.

[14] Rimas Norvaiša. Quadratic variation, $p$-variation and integration with applications to stock price modelling. Technical Report arXiv:0108090 [math.CA], arXiv.org e-Print archive, August 2001.

[15] Nicolas Perkowski and David J. Prömel. Pathwise stochastic integrals for model free finance. Technical Report arXiv:1311.6187 [math.PR], arXiv. org e-Print archive, November 2013.

[16] Nicolas Perkowski and David J. Prömel. Local times for typical price paths and pathwise Tanaka formulas. Technical Report arXiv:1405.4421 [math.PR], arXiv.org e-Print archive, May 2014.

[17] Glenn Shafer and Vladimir Vovk. Probability and Finance: It's Only a Game! Wiley, New York, 2001.

[18] Christophe Stricker. Sur la $p$-variation des surmartingales. Séminaire de probabilités de Strasbourg, 13:233-237, 1979.

[19] Kei Takeuchi, Masayuki Kumon, and Akimichi Takemura. A new formulation of asset trading games in continuous time with essential forcing of variation exponent. Bernoulli, 15:1243-1258, 2009.

[20] Vladimir Vovk. A logic of probability, with application to the foundations of statistics (with discussion). Journal of the Royal Statistical Society B, 55:317-351, 1993.

[21] Vladimir Vovk. Continuous-time trading and the emergence of probability. The Game-Theoretic Probability and Finance project, Working Paper 28, http://probabilityandfinance.com, July 2011. Journal version: Finance and Stochastics, 16:561-609, 2012.

[22] Vladimir Vovk. Rough paths in idealized financial markets. The GameTheoretic Probability and Finance project, Working Paper 35, http:// probabilityandfinance.com, arXiv:1005.0279, 2011. Journal version: Lithuanian Mathematical Journal 51:274-285, 2011. 